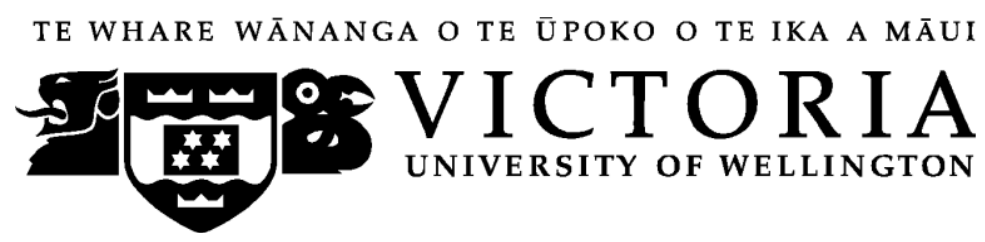

\title{
The Influence of Trust and Knowledge Sharing on Virtual Team Effectiveness
}

\section{Business Research Report}

by

Ted Thomas

(196912610)

Submitted to the Victoria University of Wellington in partial fulfilment of the requirements for the degree of Master of Business Administration

Victoria University of Wellington 2014 


\section{Acknowledgements}

I would like to acknowledge all the people who provided me with support, confidence and assistance in undertaking and completing this research.

Firstly, I thank Angela Nash, Andrew Johnston and John Campbell from QuallT for their agreement to support the research by providing access to staff across the company to participate in the survey. Without the responses from the survey, there would be no research

Secondly, I thank my supervisor, Dr Paul McDonald, without whom this work would not have progressed. He provided me with valuable direction and helped me structure this research. Also, Dr David Stewart, my Research Course co-ordinator, who offered helpful suggestions and kept me focussed on the research timeframes.

Thirdly, I thank Cheng Xhang for his guidance when I was undertaking the statistical analysis and endeavouring to gain insights from the survey responses.

Fourthly, I thank my MBA and work colleagues and friends - those who have listened to me and advised me.

Lastly, and certainly not least, I thank my wife, Mary, who has always supported me, believed in me and challenged me, throughout this MBA journey. 


\section{Table of Contents}

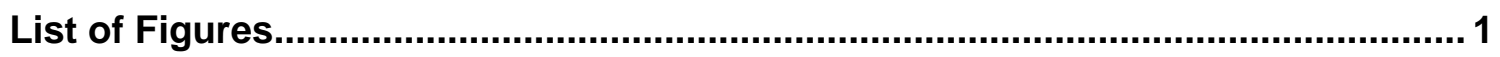

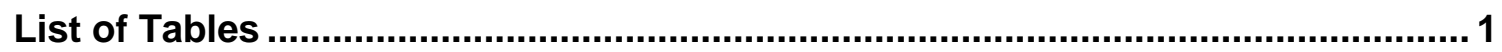

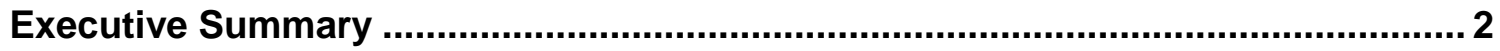

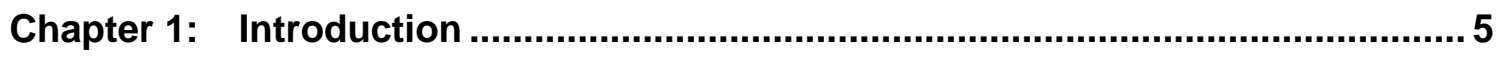

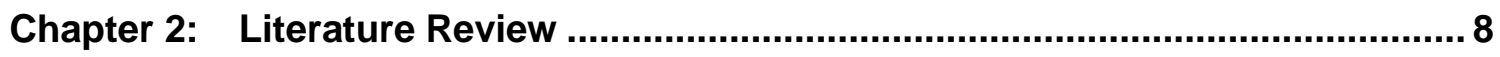

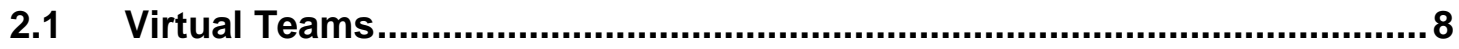

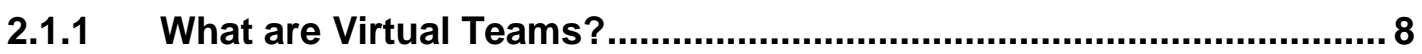

2.1.2 What has Driven the Establishment of Virtual Teams? ...................... 9

2.1.3 Comparison of Virtual with Traditional Teams..................................10

2.1.4 Advantages and Disadvantages of Virtual Teams ........................... 13

2.2 Effectiveness of Virtual Teams...........................................................15

2.2.1 What are Effective Virtual Teams? ..................................................... 15

2.2.2 What Contributes to Achieving Effective Virtual Teams? .................17

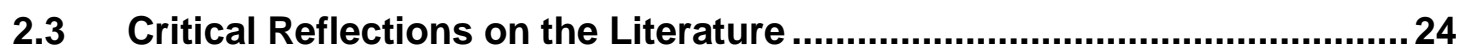

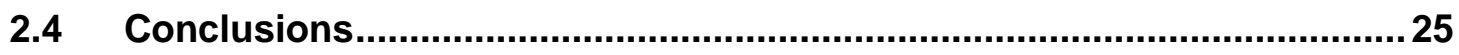

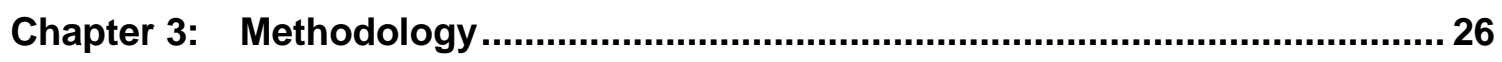

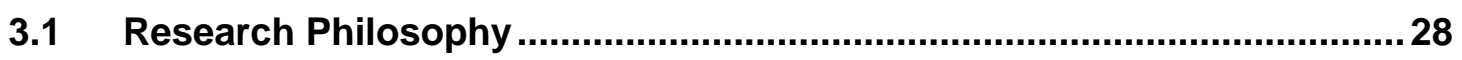

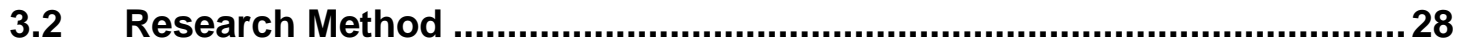

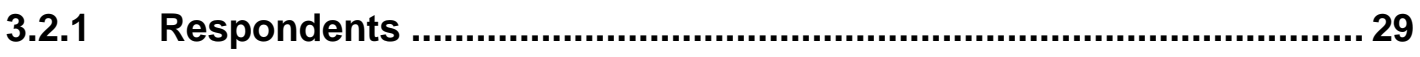

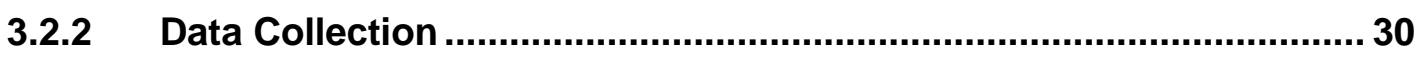

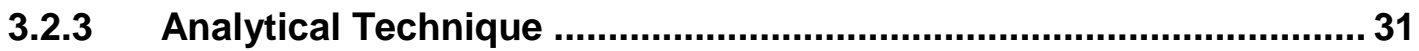

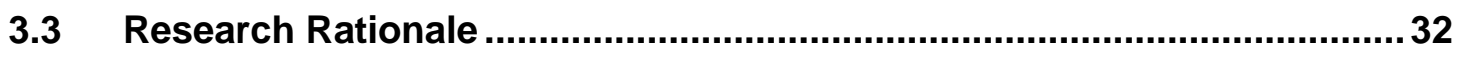

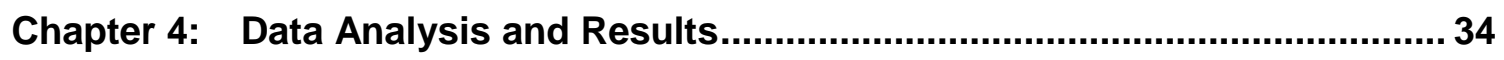

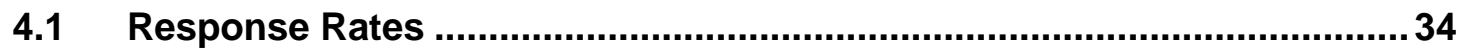

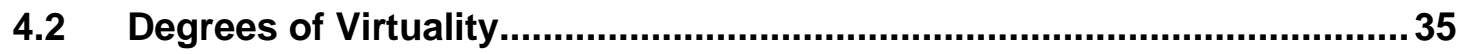

4.3 Mean, Standard Deviation and Cronbach's Alpha .................................. 35 


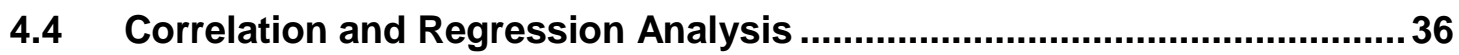

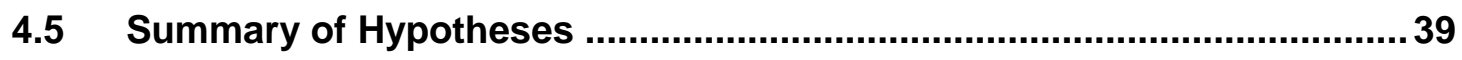

Chapter 5: Discussion and Implications ......................................................... 40

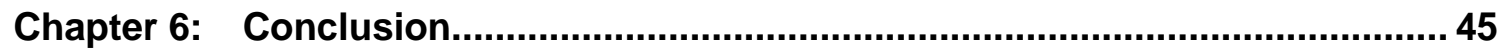

6.1 Response to the Research Question ....................................................... 45

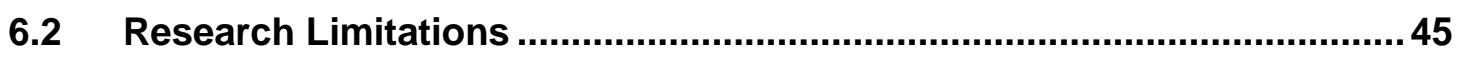

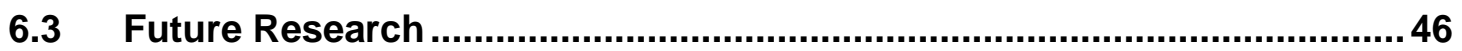

6.4 Implications for Management ..................................................................46

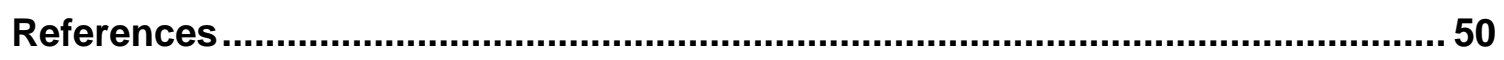

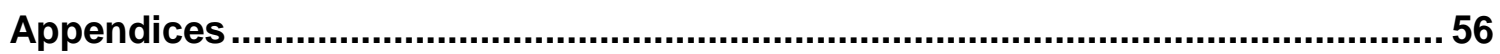

Appendix 1 - Sample Invitation Letter...............................................................56

Appendix 2 - Survey Questions ...................................................................57

Appendix 3 - Company and Customer Virtual Team Segmentation ...................61

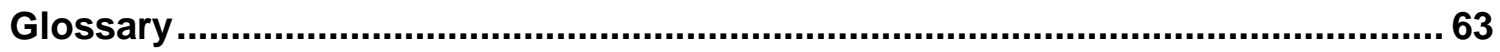




\section{List of Figures}

Figure 1: Virtual Team Effectiveness Research Framework.

Figure 2: Degree of Virtuality Response

\section{List of Tables}

Table 1: Comparing Virtual with Face to Face Teams

Table 2: Research Questions

Table 3: Derived Values

Table 4: Overview of Survey Responses

Table 5: Mean, Standard Deviation and Cronbach's Alpha Coefficient

Table 6: Pearson's Correlation of Variables

Table 7: Results of Multiple Regression - Consolidated Data

Table 7a: Results of Multiple Regression - Company Virtual Teams Data

Table 7b: Results of Multiple Regression - Customer Virtual Teams Data 


\section{Executive Summary}

Virtual teams are a growing response to increased de-centralisation and globalization, and the need for organizations to adapt to an ever changing and complex work environment. Their growing prevalence reflects many different factors, including the increased global reach of many organizations, changing workforce demographics, and heightened competitive pressures requiring greater organizational flexibility and responsiveness.

This phenomenon has grown rapidly in recent years through advancements and greater access to technologies for communication and collaboration.

Organizations however are being challenged with understanding what makes these virtual teams effective and how to measure the achievement of such effectiveness. Combined with the convergence of telephony and data technologies this has enabled voice and video to be delivered 'on demand' at a far more affordable price to the end consumer. With the added dynamic of 'mobile' becoming such a pervasive technology, this is providing the fuel driving the establishment of greater numbers of virtual teams. We now live in an increasingly "connected world" and with the blurring of work and leisure time, for many, virtual teams have already or are becoming a natural extension of the workplace.

Individuals are demanding personal flexibility in the management of their time and space and this is matched by organizations seeking flexibility to scale resources in meeting changing demand. Virtual teams may also be seen as a response to satisfying changing social and organizational aspirations.

A range of factors are seen as contributing to the effectiveness of virtual teams and these include technology, trust, sharing of knowledge, empowerment and leadership. 
This study focuses on trust as a primary factor in achieving virtual team effectiveness, and assesses the significance of trust and the sharing of knowledge amongst team members.

Trust determines how people work together, listen to one another, and build effective relationships. When people believe that they are working for trustworthy organizations, they are willing to invest their time and talents in making a difference in an organization. People who feel more connected will invest more of themselves in their work. High trust levels lead to a greater sense of self responsibility, greater interpersonal insight, and more collective action toward achieving common goals.

However, with a lack of face-to-face contact, trust based on performance substitutes for trust based on social interaction.

Trust is a cornerstone to achieving virtual team effectiveness and from an organizational perspective this highlights the need for regular communication with team members to reinforce the culture and values of the organization.

In the age of the knowledge economy, knowledge is seen as a critical resource for competitive advantage. The willingness of team members to share knowledge with others on the team can be attributed to the strength of the trust relationship and this further enhances virtual team effectiveness.

The challenges for organizations are to understand what level of trust exists across the team, how this impacts on team effectiveness and to be able to apply interventions when seeking to increase team effectiveness.

Active and regular communications programmes, internal marketing campaigns and short surveys are approaches for developing and enhancing the trust relationship. 
Organizations that are unwilling or unable to use virtual teams may find themselves losing out in an increasingly competitive and rapidly changing global economic and social environment. The technology and communication advances are clear, yet enabling effective participation and team collaboration is a more complex problem. 


\section{Chapter 1: Introduction}

The purpose of this paper is to review previous research and to extend the assessment of what primary factors contribute to achieving effectiveness of virtual teams. This introduction (chapter 1) describes the initial broad research question and why it is a topic of interest.

Virtual teams are a growing response to increased de-centralisation and globalization, and the need for organizations to adapt to an ever changing and complex environment. Their growing prevalence reflects many different factors, including the increased global reach of many organizations, changing workforce demographics, and heightened competitive pressures requiring greater organizational flexibility and responsiveness. (Cordery and Soo, 2008).

Powell, Piccoli and Ives (2004, p. 6) described virtual teams "as groups of geographically, organizationally and/or time dispersed workers brought together by information and telecommunication technologies to accomplish one or more organizational tasks." Some of these virtual teams in today's organizations consist of employees both working at home and small groups in the office but in different geographic locations. These new forms of team working environments present significant challenges to management in the context of how they communicate with and manage individuals within the virtual teams and how they measure team effectiveness and productivity.

This phenomenon has grown rapidly in recent years through advancements and greater access to technologies for communication and collaboration. According to Chhay and Kleiner (2013) new technologies have enabled the rise of virtual teams and for teammates to no longer need to occupy the same physical space to work effectively. 
Organizations however are being challenged with understanding what makes these virtual teams effective and how to measure the achievement of such effectiveness. Combined with the convergence of telephony and data technologies this has enabled voice and video to be delivered 'on demand' and at a far more affordable price to the end consumer. With the added dynamic of 'mobile' becoming such a pervasive technology, this is providing the fuel driving the establishment of greater numbers of virtual teams. We now live in an increasingly "connected world" and with the blurring of work and leisure time, for many, virtual teams have already or are becoming a natural extension of the workplace.

Most of the research into teams, team building, and team development remains focused on traditional co-located or face-to-face teams. Whilst many of the principles of management are the same, the dynamics of virtual teams with a lack of face-toface interaction places greater emphasis on communication and leadership to achieve team effectiveness and productivity. Cordery and Soo (2008) found that research into virtual teams has significantly lagged their implementation.

From the rapid development of electronic information and communication media in the past decades, distributed work has become much easier, faster and more efficient (Hertel, Geister and Konradt, 2005).

The challenge to understanding virtual teams is not so much in their form but more importantly whether they are effective and efficient structures for delivering end outcomes. Berry (2011) believes that virtual teams efficiently and effectively connect people, extend the resources and enable greater levels of productivity. The effective management of virtual teams requires knowledge and understanding of the fundamental principles of team dynamics regardless of the time, space, and communication differences between virtual and face-to-face work environments. He 
goes on to say that the effectiveness of virtual teams and resulting outcomes of virtual teamwork are dependent on the resolution of miscommunication and conflict, the development of adequate and competent roles within the team for working together, and facilitating good communication between team members. Underpinning these factors is the trust built between team members and their willingness to share knowledge.

The literature review (chapter 2) looks at the past research that has been undertaken in the field. It defines what virtual teams are and what makes these teams effective. It explores the trust relationship of team members as a contributor to team effectiveness and the influence that knowledge sharing has on achieving effective virtual teams. The aim of this chapter is to document earlier research, highlight research gaps and establish the design for the research model.

The research design (chapter 3) informs the reader about the research philosophy and methodology (survey) used to gather empirical data and test the research model. This is the basis for the data analysis presented in the fourth chapter.

The data results and analysis (chapter 4) reports the analysis of data gathered from the survey. The results of statistical analyses of the data as well as illustrative graphs and tables are presented and used as a basis for the discussion (chapter 5).

The discussion and implications (chapter 5) interprets and discusses the data analysis and then relates the findings to a comparable study and to the identified gaps in the existing literature (chapter 2).

This report concludes (chapter 6) with a summary of the key findings and qualified responses to the research questions. It also acknowledges the limitation of this research and gives recommendations for future research. 


\section{Chapter 2: Literature Review}

This literature review is designed to improve our understanding of virtual teams, how these teams operate and to gain insights into what factors contribute to virtual team effectiveness.

\section{$2.1 \quad$ Virtual Teams}

\subsubsection{What are Virtual Teams?}

Gibson and Gibbs (2006) have characterized virtual teams in terms of the geospatial distribution of their members (geographic dispersion), ranging from those that occupy the same physical space but who operate at different (non-overlapping) times to those whose members are distributed across different continents and time zones. The degree to which the team depends on electronic devices for task-related communication also varies considerably (electronic dependence), with some teams able to mix electronic interactions with regular face-to-face meetings, whereas others must interact completely via e-mail and the Internet. It has also been noted that there is an inherent dynamism about many approaches to the workings of virtual teams (dynamic structure).

Early research showed that virtual teams have played an increasingly important role in the era of globalization and provide an edifice to organizational flexibility so that it remains competitive in the global arena (Moshowitz, 1997).

Powell, Piccoli and Ives (2004, p. 6) define virtual teams in their literature review article "as groups of geographically, organizationally and/or time dispersed workers brought together by information and telecommunication technologies to accomplish one or more organizational tasks." Teams and teamwork are a ubiquitous part of getting work done in almost every organization (Hackman, 2002). Virtual team members may be located across the office, but almost as easily across the country or 
across the world, and may rarely or perhaps never meet face to face. Virtual teams are not required to use computer-mediated communication technologies, but this is typical given the near-universalistic nature of computer-mediated communication systems in organizations. Significantly, the use of technology alone does not make a team virtual. Almost all teams use technology to some degree, but virtuality increases as the degree of reliance on electronic communication increases. (Berry, 2011) Geographically dispersed teams often have no choice except to communicate electronically, even though some individual team members may strongly prefer faceto-face interaction (Cohen and Gibson, 2003). Kirkman and Mathieu (2005, p. 702) define virtuality as "the extent to which team members use virtual tools to coordinate and execute team processes." Thus, virtuality describes the degree to which teams use technology to communicate and coordinate their activities and efforts.

\subsubsection{What has Driven the Establishment of Virtual Teams?}

Many observers of macro systems, popular culture, technology, and social science name a short list of global trends-including digitalization, globalization, value pluralism, and climate change, among other significant social trends, that are altering societies at an accelerating pace. These macro trends also are changing the nature of collaborative efforts—at work or anywhere else—by altering the opportunities for collaboration, the need to build collaborations, and the structure of collaborations when they arise (Wageman, Gardner and Mortensen, 2012). With the continued march of globalization, more people are collaborating across nationalities, cultures, and languages as well as across space and time.

Virtual teams are made possible by a proliferation of fibre optic technology that has significantly increased the scope of off-site communication (Vlaar, 2008). An example of this can also be seen through social media and where technologies like Facebook, Twitter and Linkedln represent virtual spaces that are creating vast virtual 
communities and teams. One might say that virtuality has now become a significant part of our daily lives and the challenge will be to adapt and how to operate within this continually advancing new paradigm.

Firms are increasingly using virtual teams to accomplish complex tasks, staffing them with the best people for the job irrespective of location (Chiravuri and Nazareth, 2011). The primary advantage of virtual teams is flexibility and dynamism, so organizations can respond rapidly and effectively to continual (and often unforeseen) changes in the environment (Suchan and Hayzak, 2001).

In the wake of global expansion and outsourcing, organizations are working hard to cut the cost and minimize the hassles of bringing team members to a single location. Hence, the virtual team structure was adopted to solve this problem. Furthermore, this type of structure is also attractive to most employees because the trend among the new generation of workers is to demand personal flexibility. Therefore, it is important to know how to make virtual teams more effective (Nunamaker, Reinig and Briggs, 2009). Despite research done in the past, there is still uncertainty in relation to factors that contribute to virtual team effectiveness (DeRosa, 2009; Lin, Standing and Liu, 2008).

\subsubsection{Comparison of Virtual with Traditional Teams}

Griffith \& Neale (2001) conclude that any team is virtual to some extent at least some of the time. Traditional or face-to-face teams, for example, may use an electronic medium to send out minutes from a meeting or even to confirm decisions reached during a hallway chat. Considering teams along this continuum from only virtual to only face to face is appealing because most teams utilize some combination of faceto-face and computer-mediated communication in their interactions. 
Berry (2011) described most problems in virtual teams evolving from a lack of personal willingness of team members to participate, lack of general planning by the team or management, conflicting schedules, or the common issue of individual disagreements, as much as within face-to-face teams. Significantly, most of these interaction issues can be resolved in the virtual environment although not in the same ways as they are resolved in the face-to-face environment. Despite all the complications and confounding characteristics of virtual as compared to co-located face-teams, effective and trusting virtual teams can be built. Trust based on performance substitutes for trust based on social interaction.

Communication, whether virtual or face to face, is fundamental to getting any organizing or work done, as communication provides the basic building blocks with which people collaborate, make decisions, and act to achieve organizational objectives. Communication is also central to organizational socialization including sense making and affiliation (Flanagin and Waldeck, 2004).

Johnson, Chanidprapa, Yoon, Berrett, and LaFleur (2003) found that virtual team members were less inhibited because of not being co-located and that ideas and feedback in the virtual environment were more frank.

Research suggests that the skill sets required for success in managing effective virtual work teams are more complex than the skill sets required for success in managing face-to-face teams. Age differences (in the matter of professional commitment) and gender differences (social constraints, sense of affiliation, and cognitive disparity) play a significant role in determining virtual team effectiveness (Gaan, 2012). 
Berry (2011) produced a comparative summary of team attributes highlighting aspects of team membership, communication/interaction, teamwork and trust as represented in Table 1 below. This provides some good insights into what are likely to be different team dynamics.

\section{Table 1: Comparing Virtual with Face to Face Teams}

\section{VIRTUAL TEAMS}

Team Members:

Can be located anywhere in the world

Are from an open-ended source of experts

Have often not worked together before

Communication / Interaction:

Usually computer-mediated communication, seen as different

Is usually asynchronous; slower

Usually a pause before response

Responses often more reflective and data driven because researched

Teamwork:

Has little social interaction

Is mostly task focused

Performance is equivalent to face-to-face teamwork, over time

Trust on the Team:

Initially likely based on task performance

Over time, usually data-/proven

performance based

Usually develops more slowly than trust on face-to-face teams

\section{FACE-TO-FACE TEAMS}

Team Members:

Are usually co-located

Are from a very limited source of experts

Usually have worked together before

Communication / Interaction:

Usually face-to-face, and this is seen as the norm, taken for granted, and best

Synchronous, instant

Responses usually immediate

Responses often superficial because off-the-cuff, not researched

Teamwork:

Opportunity for much social interaction

Blend of social and task focus

Performance is equivalent to virtual team performance, over time

Trust on the Team:

Initially likely based on social or personal characteristics

Over time, usually a blend of social and task performance-based

Usually develops more quickly than trust on virtual teams 


\subsubsection{Advantages and Disadvantages of Virtual Teams}

\subsubsection{Advantages of Virtual Teams}

Berry (2011) contends that an advantage of virtual teams is that team members are able to communicate, collaborate, and create outputs irrespective of time and space, because they are not bound by temporal constraints or geographic location as are most face-to-face teams. Virtual teams enable organizations to attain a broader geographic reach whilst maintaining effective contact with employees and customers (Maynard, 2006).

Pangil and Chan (2014) concluded that there are various reasons that lead organizations to adopt virtual teams. Some of these reasons are:

- to be able to hire the best employees who may be located anywhere in the world;

- to increase the global workday to 24 versus 8 hours;

- to provide flexibility to support the globalization of trade and corporate activity in order to be more competitive and responsive to the marketplace.

Vroman and Kovachich (2002) identified one advantage of having virtual teams is that different opinions and perspectives are represented within the team and thus greater organizational learning and synergy are possible because of this added diversity. Shared goals and shared understandings are required on any team, and negotiation of these common goals is an intrinsic part of the team-building process.

Performance is often easier to document and review in virtual teams because most interactions, commitments, and outcomes are archived automatically and electronically (Gibson and Cohen, 2003). Asynchronous processes are often more efficient because participation occurs in parallel instead of serially (with attendant 
communication blocking) as with most communication on face-to-face teams (Klein and Kleinhanns, 2003).

Kirkman and Mathieu (2005) argue that with the advances in technology and increases in team members understanding and usage of technological capabilities, these media can now be used advantageously to enhance team functioning. An example of this was the $\$ 1$ million winners of the 2009 Netflix innovation award. The seven-member team that developed an algorithm to improve online recommendations met physically for the first time when they received their reward (The Economist, 2010).

\subsubsection{Disadvantages of Virtual Teams}

Working in a virtual environment, teams have a lower frequency of face-to-face contact and this has proven to be a hurdle to knowledge sharing and the development of trust across team members. Research indicates that achieving this simply takes more time. Negative outcomes are clearly possible when using virtual teams. Hinds and Weisband (2003) found that virtual team members tend to initially share less information than members of face-to-face teams. Thus, team members may have weaker shared understandings of needed outcomes, which in turn may cause negative effects on performance outcomes; however, given time these lack-ofshared-information issues appear to fade (Walther, 1995). These issues and problems are real at least in the short term, but they tend to fade as team members become more experienced with the logistics or systems of the virtual workplace. Over time cohesion and satisfaction does form in virtual teams although this cohesion seems to take longer than in co-located teams (Burke, Aytes and Chidambaram, 2001). 
The absence of physical presence is considered by some to be the major drawback of virtual teams and virtual work (Cohen and Gibson, 2003). Some virtual team members may be less productive or satisfied because they feel isolated and detached from both the work and the other team members. Indeed, the literature on motivation and satisfaction holds that most employees are motivated and satisfied in part as a result of interactions with co-workers (Kirkman and Mathieu, 2002).

\subsection{Effectiveness of Virtual Teams}

\subsubsection{What are Effective Virtual Teams?}

Literature shows that there are many factors that have been associated with the effectiveness of teams, however very few studies focus specifically on virtual team effectiveness. Nonetheless, these few studies have shown that some of the factors that were associated with virtual team effectiveness include the shared mental model between the team members (Xiao and Jin, 2010), trust (Kanawattanachai and Yoo, 2007; Sarker, Valacich and Sarker, 2003), control structures (Powell, Piccoli and Ives, 2004), and communication effectiveness (Powell, Piccoli and Ives, 2004).

Pangil and Chan (2014) described "to be effective" as being the objective of any individual, team or organization in performing their tasks. However, effectiveness is a subjective term and difficult to define and measure. Hence, it is important to be clear about the dimension of effectiveness that is being considered and the levels at which they are being considered. This is because effectiveness as defined and measured at the individual level is different from effectiveness at the team or organization level. Nevertheless, effectiveness at one level of analysis would still affect the effectiveness at another level.

Cordery and Soo (2008) proposed that, compared to traditional teams, virtual teams are particularly challenged when it comes to (a) accessing, sharing, and capitalizing 
on team knowledge; (b) developing a sense of collective engagement in respect of the team task; and (c) experiencing the sense of collective competence that is often associated with performance excellence. The conceptual model is one in which typical attributes of virtual teamwork are linked to effectiveness indicators by means of three key mediating team processes - transactive memory, work engagement, and collective efficacy. The existence of a strong transactive memory enables team members to understand what knowledge is possessed by the team, who to ask for information, who is the best person for a particular job, and who is more likely to understand certain types of information as it enters the team. The motivational aspect of work and the significance of intrinsic satisfaction were identified as a major challenge.

Research has indicated that work engagement may be experienced not just by individuals but at a collective or team level, and have a powerful effect on team-level outcomes, such as performance and member satisfaction (Salanova, Agut and Peiro, 2005).

Collective efficacy refers to the strength of a team's beliefs that it has the capability to perform effectively. That is, group efficacy forms as group members collectively acquire, store, manipulate, and exchange information about each other and about their task, context, process, and prior performance" (Gibson, 1999). Effective teams need to develop the knowledge structures, motivation, and performance beliefs that ensure that they perform effectively.

Research conducted by Gera, Aneeshkumar, Fernandez, Gireeshkumar, Nze and Eze (2013) concluded that successful virtual organizations are those wherein collaboration, cohesion and trust takes place among individuals. 


\subsubsection{What Contributes to Achieving Effective Virtual Teams?}

Despite research having been undertaken in the past in relation to virtual team effectiveness (Kanawattanachai and Yoo, 2007; Lin, Standing, and Liu, 2008; Ross, Jones and Adams, 2008), there is still uncertainty of what these factors are. The literature however is quite broad in its discussion on contributing factors and it may be more accurate to say that there is a lack of clarity as to which factors (or combination of factors) are the major contributors to achieving effective virtual teams. When we consider that we are dealing with human behaviours and interactions, this adds complexity to assessing "effectiveness" as an outcome of virtual team achievement.

From the literature reviewed, a range of factors contributing to the effectiveness of virtual teams have been identified as technology, trust, sharing of knowledge, empowerment and leadership. These factors are discussed further below.

\subsubsection{Technology}

Although technology's function in the virtual team enables the completion of work and overcomes many of the complexities created by time and distance, these technologies still need to be understood as only a communication and collaboration tool and not as communication or collaboration itself. (Berry, 2011)

The basic condition for effectiveness of a virtual team is the provision of effective communication between its members (Dustdar, 2004). One of its aspects is constituted by a phenomenon of social presence, describing the sense of belonging to a group, awareness of other people's presence as well as the possibility of making easy and quick contact (Hakkinen, 2004). 


\subsubsection{Trust}

Simply stated, trust is the belief or confidence in another person or organization's integrity, fairness, and reliability (Berry, 2011).

Based on the work of Sarker, Valacich and Sarker (2003), trust within virtual teams can be defined as the degree of reliance that individuals have on their remotely located team members taken collectively (as a group). In total there are three dimensions in defining virtual team's trust, i.e. personal-based, institutional-based, and cognitive-based trust. Personal-based trust is the type of trust that develops during childhood. It develops based on the nurturance that one received from one's caretaker, and it has a lasting effect and can be seen in individual's everyday interaction with others (Sarker, Valacich and Sarker, 2003). Hence, this type of trust is innate to one's personality and individuals that demonstrate these attributes of trust would be seen as trustworthy persons.

On the other hand, institutional-based trust is the type of trust that exists when one conforms to the rules and regulations of an organization. It develops because organizations demand it from its members or the members' fear of the repercussions of not conforming to the needs and requirements of the organization (Sarker, Valacich and Sarker, 2003). Institutional based trust is important for virtual team effectiveness because such teams need people who are self-monitoring, and are afraid of the negative consequences that could result for not completing their tasks. Such individuals will be able to regulate their own behaviours to always meet the demands and requirements of the organization.

Cognitive-based trust develops during one's interaction with one another. It is based on schemas that individuals develop about their team members (Sarker, Valacich and Sarker, 2003). This type of trust is also difficult to develop within virtual teams 
because of the limitations inherent in virtual teams. Nonetheless, developing such trust among the members will be most beneficial to team effectiveness, and the findings of Kanawattanachai and Yoo (2007) showed that cognitive trust is significantly related to virtual team performance.

Zhang, Fang, Wei, and Chen (2010) argued that not only trust is of high importance in ensuring the effectiveness of virtual teams, but also the occurrence of knowledge sharing between virtual team members. Pangil and Chan (2014) concluded that knowledge sharing and trust had a significant bearing on virtual team effectiveness.

\subsubsection{Sharing of Knowledge}

In the age of the knowledge economy, knowledge is seen as a critical resource for competitive advantage. Hence, it is crucial that organizational knowledge is managed diligently. One important aspect of managing knowledge is to encourage individuals within the organization to share knowledge with other members of the organization (Davenport and Prusak, 2000).

In recent years, organizations have relied on mobilizing more diverse sets of unevenly distributed knowledge resources through virtual teams. Organizations established virtual teams with the purpose of combining the expertise of individuals that are located in different places (Cohen and Bailey, 1997). It is to enable knowledge sharing and transfer to occur as a path to gaining competitive advantage. If organizations are able to promote knowledge sharing within virtual teams, not only could they fully utilize the knowledge that exists within the organization but also enhance the value of knowledge because the dialogues involved during sharing often lead to the generation of new ideas, which is considered as having the potential for the creation of new knowledge (Nonaka, 1994). In fact, shared knowledge among 
virtual team members has been shown to enhance the performance of the teams (Xiao and Jin, 2010).

Virtual team members should be able to effectively share their knowledge due to their mutual influence, mutual commitment and mutual conflict (Wu, Lin, and Lin, 2006). However, effective knowledge sharing between members is more difficult in virtual teams than in traditional forms of teams. For knowledge sharing to occur, trust among team members is essential (Abrams, Cross, Lesser, and Levin, 2003; Holste and Fields, 2010). In addition to that, when a new virtual team is assembled for the first time, research indicates that it takes a few weeks before the members are able to fully recognize, trust, and coordinate their specialized knowledge in order to effectively perform the task (Kanawattanachai and Yoo, 2007). Hence, trust is also essential for knowledge sharing to occur among virtual team members and this will eventually heighten the performance of the team.

Naturally, people will be more willing to share knowledge with others who by nature can be trusted, and hence personal-based trust is essential in virtual teams. However, members do not get to see each other regularly and sometimes not at all, and therefore personal-based trust is rather difficult to establish. Due to this, institutional-based trust becomes an imperative for knowledge sharing among virtual team members. Finally, studies have shown that cognitive-based trust is significantly related to knowledge sharing (Holste and Fields, 2010). In essence, it is the perceived competence and professionalism that each member has of the others that enhances his or her willingness to share knowledge.

Pangil and Chan (2014) developed a framework for research conducted to establish the relationship between trust and knowledge sharing as a basis for determining 
virtual team effectiveness. They concluded that "knowledge sharing is important regardless of whether the team is conventional or virtual in nature"

\subsubsection{Empowerment}

Team empowerment is a shared motivational state that incorporates elements of both work engagement (perceived task meaning and importance) and collective efficacy (potency), and is generally recognized as being strongly influenced by external team leadership (Mathieu, Gilson and Ruddy, 2006).

Arnold, Arad, Rhoades and Drasgow (2000) identified five key dimensions of leader behaviour linked to team empowerment. These are leading by example, participative decision making, coaching, informing, and showing concern for/interacting with the team.

Dube and Robey (2008) suggest that since monitoring member activities is often difficult in virtual teams, empowerment could improve performance. If managers of virtual teams coordinated team activities rather than directed them, they could be more effective. An empowered and empowering manager will have characteristics of a democratic power orientation, low levels of involvement, self-regulates, is a boundary spanner, and helps team members in any way necessary (Oakley, 1998). 


\subsubsection{Leadership}

Whilst the establishment and use of virtual teams has grown rapidly, one might say by stealth, there has been relatively little focus on the role of the leaders of virtual teams and their influence in achieving effective virtual teams.

Research on team leadership in general has largely focused on individuals who occupy leadership roles. Little attention has been given to leadership teams; that is, groups of leaders who are collectively responsible for leading a social system and each of whom is himself or herself a significant organizational leader. (Wageman, Gardner and Mortensen, 2012). Researchers have been quick to recognize the important role played by team leaders and managers in supporting virtual teams (DeRosa, Hantula, Kock and D'Arcy, 2005).

More organizations are encouraging self-forming, self-governing teams who define their own purposes, choose their own members, and design their own work (Nonaka and Toyama, 2005). Teams are increasingly replacing individuals in leading themselves and their organizations. (Wageman, Gardner and Mortensen, 2012).

Virtual teams have given rise to a new set of leadership challenges especially as it relates to all-important team outcomes and performance (Hambley, O'Neill and Kline, 2007). In addition, what is presupposed in the virtual team leadership research is that the teams have assigned leaders. In many instances, virtual team leaders emerge through growing influence and contributions to the team (Yoo and Alavi, 2004).

Whilst technology helps connect teams, research showed that greater distances increase coordination delays, although time is a greater barrier than physical distance. (Chhay and Kleiner, 2013) 
Hoegl and Siebdrat (2009) suggest that the performance of teams suffers with increasing levels of dispersion. Because of that, managers have typically viewed dispersion as a liability rather than an opportunity. But dispersion can provide substantial benefits if organizations can take advantage of the diversity and varied expertise of team members at different locations. They identified the following key issues when managing virtual teams:

- Don't underestimate the significance of small distances.

- Emphasize teamwork skills.

- Promote self-leadership across the team.

- Provide for face-to-face meetings.

- Foster a "global culture." 


\subsection{Critical Reflections on the Literature}

Based on the literature and from professional experience, the trust relationship established between team members and the trust bestowed upon teams by the organization itself, create working environments based on mutual respect. Within this virtual team construct there are performance expectations set that translate into measures of virtual team effectiveness. Time must also be a key factor in the development of trust relationships (the elapsed time required to develop trust) and a direct bearing on the effectiveness of virtual teams. Trust takes time to develop and mature amongst team members and it is through regular communications and interactions that this occurs. Face-to-face communication between team members is often part of this process and by gaining an understanding of the elapsed time associated with establishing and developing virtual teams, and the process of constructing virtual teams, we may gain a better appreciation of the factors that contribute to virtual team effectiveness.

The rapidly changing technologies used for communication and collaboration within virtual teams are providing greater transparency of team operations and are indicators contributing to team performance. It is quite likely that this is also having a positive impact on enhancing trust amongst team members and as a consequence improving the effectiveness of virtual teams. These same technologies are facilitating both formal and informal team member communications and providing a platform for nurturing personal and institutional-based trust.

The culture of organizations embracing virtual teams is something not explored in depth within the literature and may be an area to better understand in terms of the organizational support needed for virtual teams. 


\subsection{Conclusions}

Organizations that are unwilling or unable to use virtual teams may find themselves losing out in an increasingly competitive and rapidly changing global economic and social environment. The technology and communication advances are clear, yet enabling effective participation and team collaboration is a more complex problem.

The review of the literature on the effectiveness of virtual teams yielded three conclusions.

The first conclusion is that effective virtual teams are achieved through effective organizational and team leadership.

The second conclusion is that effective virtual teams require the right information technology and communications collaboration infrastructure to be in place and accessible to team members.

The third conclusion is that the empowerment of virtual teams builds trust amongst team members and a willingness to share knowledge, resulting in higher levels of effectiveness in virtual teams. 


\section{Chapter 3: Methodology}

This chapter introduces the research philosophy, that is, the position the researcher is taking on this research. The research method is established and the research analytical technique is introduced. This chapter concludes with the rationale for conducting the research.

Based on the review of the literature, three research questions emerged and are listed below in Table 2:

\section{Table 2: Research Questions}

\begin{tabular}{|c|l|}
\hline 1 & $\begin{array}{l}\text { Does the trust relationship between team members } \\
\text { contribute to achieving effective virtual teams? }\end{array}$ \\
\hline 2 & $\begin{array}{l}\text { Does the trust relationship between team members } \\
\text { increase the willingness to share knowledge? }\end{array}$ \\
\hline 3 & $\begin{array}{l}\text { Does the sharing of knowledge between team members } \\
\text { enhance virtual team effectiveness? }\end{array}$ \\
\hline
\end{tabular}

There are three main variables identified in this study. The dependent variable is virtual team effectiveness, trust within the team is the independent variable, and knowledge sharing is a mediating variable. The trust variable consists of three dimensions; i) personal-based trust (PBT), ii) institutional-based trust (IBT), and iii) cognitive-based trust (CBT). The relationships of the variables are depicted below in Figure 1.

Figure 1: Virtual Team Effectiveness Research Framework

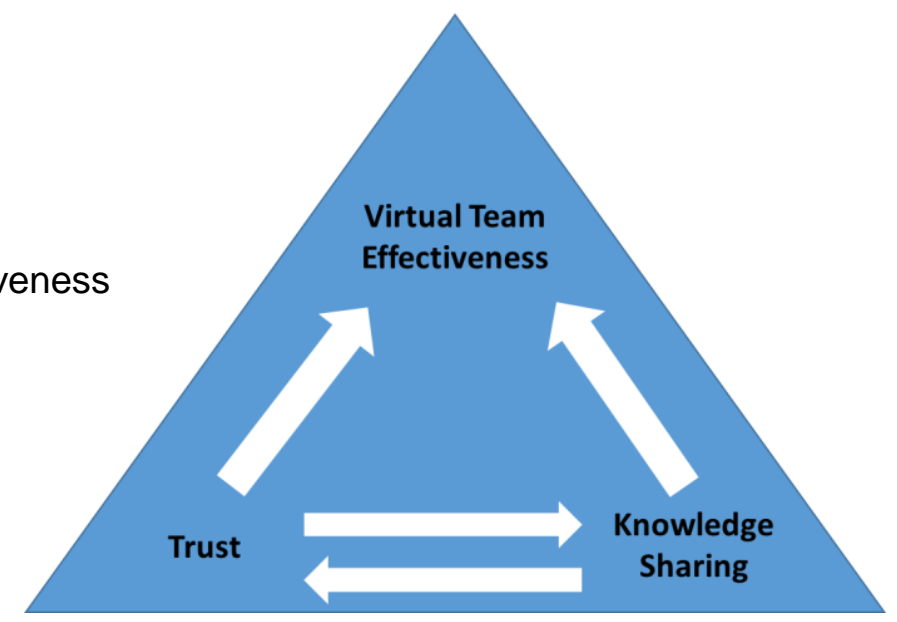


In order to test the mediating effects of knowledge sharing, there is a need for evidence that there is a significant relationship between trust and virtual team effectiveness, trust and knowledge sharing, and finally knowledge sharing and virtual team effectiveness (Baron and Kenny, 1986). Based on these requirements, it is hypothesized that:

Hypothesis $1(\mathbf{H} 1)$ There is a significant positive relationship between trust (PBT, IBT, and CBT) and virtual team effectiveness, with CBT presenting the strongest correlation.

Hypothesis 2 (H2) There is a significant positive relationship between trust (PBT, IBT, and CBT) and knowledge sharing, with PBT presenting the strongest correlation.

Hypothesis $3(\mathbf{H} 3)$ There is a significant positive relationship between knowledge sharing and virtual team effectiveness.

Hypothesis $4(\mathbf{H} 4)$ Knowledge sharing mediates the relationship between trust (PBT, IBT, and CBT) and virtual team effectiveness.

This study focuses on trust as a primary factor and objectively assesses the significance of trust amongst team members and the sharing of knowledge in achieving virtual team effectiveness.

The research is based on a study conducted by Pangil and Chan (2014) where they examined the relationship between the three dimensions of trust and virtual team effectiveness, and went on to investigate the role of knowledge sharing as a mediator to this relationship. 


\subsection{Research Philosophy}

This research project is conducted from a positivist perspective and that is it describes the phenomena experienced through observation and measurement. It is important that the data collected and analysed is as objective as possible and that it truly reflects what is being observed to reliably extend our understanding of what influences virtual team effectiveness. Driscoll (2000) states that reality is external and is objective, and knowledge is gained through experiences. Positivism is founded on cause and effect relationships and outcomes. This research focuses on observing trust and knowledge sharing as behaviours (causes) that impact on virtual team productivity and effectiveness (effect).

Reliability of the research is very important in terms of its measurement and must be replicable if it is to stand up to external scrutiny. As in many positivist studies, a quantitative approach was chosen and whilst the survey was from a single source company, as large a sample as possible was collected in order to obtain statistically significant findings that can be generalized across the population studied.

Positivist research findings are not biased by the researcher and his or her beliefs, as the researcher is independent of the situation being studied.

\subsection{Research Method}

A quantitative approach was used to collect data in a cross-sectional anonymous manner with the use of a survey questionnaire. Qualtrics, a web-based survey tool was used to create and host the questionnaire and an email was sent to potential participants with a link to the survey. The email was in the form of a personal invitation to participate in the survey and was sent from the Chief Executive of QuallT, a Wellington, New Zealand headquartered information technology testing services company. The survey was open for a fixed period of 14 days and two follow 
up emails were issued over the survey period to all invitees (including those that may have already responded) reminding them to complete the survey and in an attempt to drive and increase the response participation.

\subsubsection{Respondents}

QuallT (http://www.qualit.co.nz), agreed to participate in the research and provide access to their staff for the purpose of conducting the survey. QuallT have offices in Wellington, Auckland and Christchurch, employ approximately 170 staff and operate virtual teams of software testers. These teams tend to be project based meaning that the makeup and dynamics of the teams are changing regularly. Senior management of the company also operate a virtual executive team across the three company office locations. The survey was conducted with 140 invitees, all being QuallT staff and with the majority spending their time working within virtual teams.

Because the survey was anonymous, it was impossible to know who had responded and who had not, and therefore blanket emails to all participants were sent out as reminders. The survey remained online for two weeks with each respondent being asked to complete all questions. Functions provided by the Qualtrics survey tool prevented participants from completing the survey more than once and validation enforced completion of all questions within a survey session (except where the respondent chose to force close the survey).

The management of QuallT intend to provide all survey invitees with a summary copy of the research report as feedback and communication highlighting the factors attributed to virtual team effectiveness and the team trust relationships that drive these outcomes. 


\subsubsection{Data Collection}

The questionnaire required the respondents to answer questions regarding three different types of trust (personal-based trust, institutional-based trust and cognitivebased trust), their knowledge sharing behaviour and an evaluation of the effectiveness of the virtual teams that they worked in. The following areas were surveyed and details of the survey questions can be referenced in Appendix $2-$ Survey Questions.

1. Measuring virtual team effectiveness - The questions are adopted from a study conducted by Lurey and Raisinghani (2001). This contains nine questions and it captures and quantifies the level of team performance and satisfaction with regard to the team.

2. Measuring the three types of trust - - The questions were adopted from a study conducted by Sarker, Valacich and Sarker (2003). This section contained twenty eight questions, consisting of four questions for measuring personal-based trust, seven questions for measuring institutional-based trust and seventeen questions for measuring cognitive-based trust.

3. Measuring the effect of trust on knowledge sharing - The questions were adopted from a study conducted by Staples and Webster (2008). This contains five questions for measuring the effect of trust on knowledge sharing.

The "degree of virtuality" was established with respondents being asked to attribute the amount of time (as a \%) usually spent working in a virtual team environment.

Respondents were also asked to classify the type of virtual team they currently worked in as being either a company (QuallT) or customer virtual team. This additional segmentation of respondents was helpful when testing the responses 
relating to the trust relationships and provided insights into whether the virtual team (company or customer) had an impact on virtual team effectiveness.

The survey was piloted initially with a small number of users external to QuallT to test usability and to gain feedback in advance of full rollout. The pilot testers were asked to check for any ambiguous or misleading questions which could have led to misinterpretation and the introduction of biased or invalid data. The survey took on average between 10-15 minutes to complete.

Robustness of the survey questionnaire in terms of the questions asked, the number and quality of responses were risks sought to be mitigated by receiving the active endorsement of the QuallT Chief Executive and support of the senior management team. Reliability and validity of the data collected were important criterion that underpinned the credibility of the research.

\subsubsection{Analytical Technique}

Analysis was employed to identify the strength of relationships between trust and knowledge sharing (independent variables) and virtual team effectiveness (the dependent variable).

Responses to questions 3 through 9 were measured using a seven point likert scale, with 1 representing "strongly disagree" through to 7 representing "strongly agree". Each of these questions had a series of items which for the purpose of this survey all held an equal weighting. 
Derived values were calculated from the responses for each item by summing the item responses and mapping them across a value range back to the 1 to 7 likert scale. The derived value calculations and mappings are referenced in Table 3.

\begin{tabular}{|c|c|c|c|c|c|c|c|c|}
\hline & Variables & $\begin{array}{l}\text { Virtual Team } \\
\text { Effectiveness }\end{array}$ & $\begin{array}{c}\text { Trust - } \\
\text { Personal } \\
\text { Based }\end{array}$ & $\begin{array}{c}\text { Trust - } \\
\text { Institutional } \\
\text { Based }\end{array}$ & $\begin{array}{c}\text { Trust - } \\
\text { Cognitive } \\
\text { Message } \\
\text { Based }\end{array}$ & $\begin{array}{c}\text { Trust - } \\
\text { Cognitive } \\
\text { Behaviour } \\
\text { Based }\end{array}$ & $\begin{array}{l}\text { Trust - } \\
\text { Cognitive } \\
\text { Reputation } \\
\text { Based }\end{array}$ & $\begin{array}{c}\text { Knowledge } \\
\text { Sharing }\end{array}$ \\
\hline & Questions & Q3 & Q4 & Q5 & Q6 & Q7 & Q8 & Q9 \\
\hline & Items & 9 Items & 4 Items & 7 Items & 9 Items & 4 Items & 4 Items & 5 Items \\
\hline Likert Scale & Values & Range & Range & Range & Range & Range & Range & Range \\
\hline 1 & Strongly Disagree & $0-9$ & $0-4$ & $0-7$ & $0-9$ & $0-4$ & $0-4$ & $0-5$ \\
\hline 2 & Disagree & $10-18$ & $5-8$ & $8-14$ & $10-18$ & $5-8$ & $5-8$ & $6-10$ \\
\hline 3 & Somewhat Disagree & $19-27$ & $9-12$ & $15-21$ & $19-27$ & $9-12$ & $9-12$ & $11-15$ \\
\hline 4 & Neutral & $28-36$ & $13-16$ & $22-28$ & $28-36$ & $13-16$ & $13-16$ & $16-20$ \\
\hline 5 & Somewhat Agree & $37-45$ & $17-20$ & $29-35$ & $37-45$ & $17-20$ & $17-20$ & $21-25$ \\
\hline 6 & Agree & $46-54$ & $21-24$ & $36-42$ & $46-54$ & $21-24$ & $21-24$ & $26-30$ \\
\hline 7 & Strongly Agree & $55-63$ & $25-28$ & $43-49$ & $55-63$ & $25-28$ & $25-28$ & $31-35$ \\
\hline
\end{tabular}

Pearson's correlation analysis is performed to determine the relationships between trust, knowledge sharing and virtual team effectiveness. This is followed by hierarchical regression analysis to test the mediating effect of knowledge sharing on the relationship between trust and virtual team effectiveness.

The results of this analysis tested the support for the hypotheses outlined in the Research Method above to determine the strength of correlation between the factors as contributors to achieving virtual team effectiveness.

\subsection{Research Rationale}

Trust and virtual team effectiveness present a complex relationship at the heart of the performance of virtual teams. It is crucial that organizations gain a better understanding of this relationship for them to be in a stronger position to manage virtual teams and their team members with the objective to positively influence the achievement of virtual team effectiveness. 
In facing rapid advancements in technology to support virtual teams and increased demand from employees to participate in these type of teams, management has the choice of either determining the structures and outcomes that they want from these virtual environments or to allow the virtual environments to evolve and in so doing, reduce the ability to control and/or influence the behaviours.

The pace of change in the area of communication and collaboration technologies has shown that whilst earlier studies focused on virtual teams' performance and effectiveness, they are quickly losing their relevance having been benchmarked using quickly dating technologies. 


\section{Chapter 4: Data Analysis and Results}

This chapter gives an overview of the survey responses and examines the questions used in the survey.

\subsection{Response Rates}

A total of 140 QuallT staff were invited to participate in the survey and 57 responses were started, representing a $40.7 \%$ response rate. Of the 57 survey responses started, 40 (70.2\%) were completed as represented in Table 4 below. This response was considered an acceptable representative number of responses considering the tightly controlled and targeted participant group surveyed.

\begin{tabular}{|l|c|c|}
\hline \multicolumn{3}{|l|}{ Table 4: Overview of Survey Responses } \\
\hline Category & Responses & $\%$ \\
\hline Sent & 140 & $100 \%$ \\
\hline Started & 57 & $40.7 \%$ \\
\hline Completed/Sent & $40 / 140$ & $28.6 \%$ \\
\hline Completed/Started & $40 / 57$ & $70.2 \%$ \\
\hline
\end{tabular}




\subsection{Degrees of Virtuality}

$82.5 \%$ of respondents identified themselves as working in a virtual mode more than $60 \%$ of the time. This enhances the reliability of the data in knowing that a high percentage of the responses to the questions are from staff who are primarily engaged in virtual teams and experience the trust relationships and knowledge sharing as a normal part of their daily working environments.

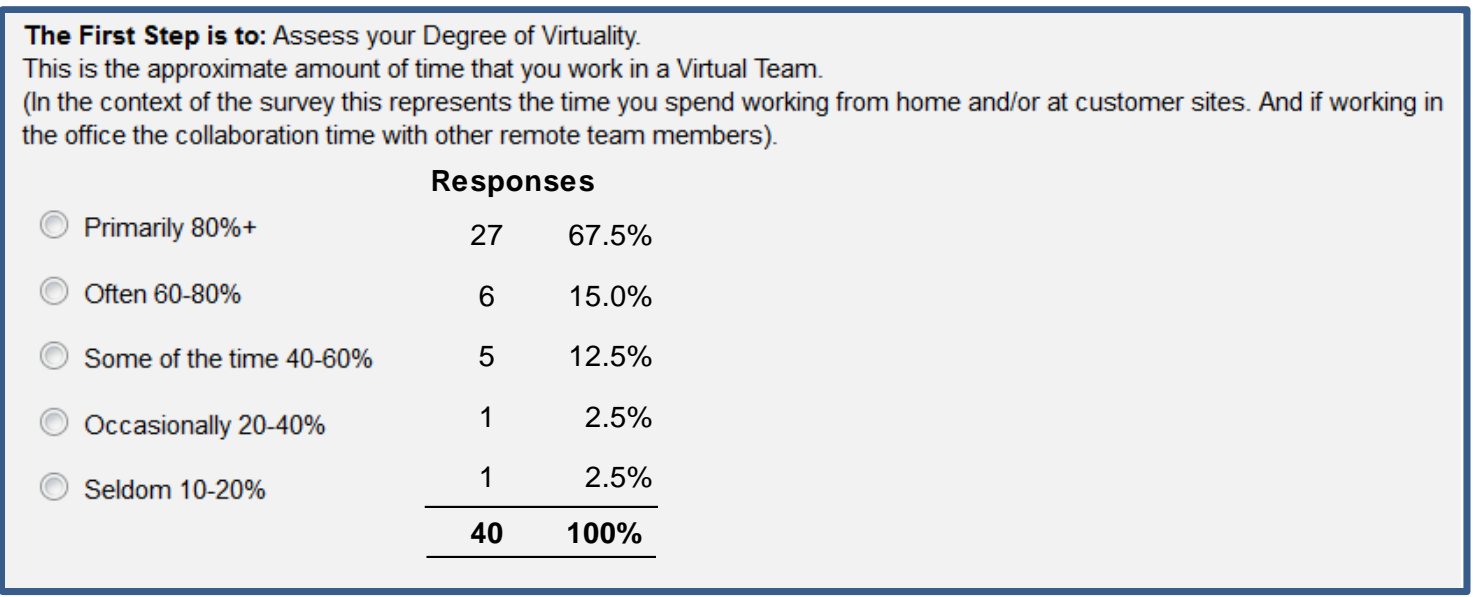

Figure 2: Degrees of Virtuality

\subsection{Mean, Standard Deviation and Cronbach's Alpha}

The mean, standard deviation and Cronbach's alpha coefficients were calculated for each question, with the results provided in Table 5. Cronbach's alpha coefficient assesses the internal consistency of the items of each question and provides an estimate of the reliability of the items from the responses to the questions. The rule of thumb is that the alpha value should be greater than 0.7 and this is supported from the survey responses with the alpha values ranging from 0.8392 to 0.9428 .

\section{Table 5: Mean, Standard Deviation \& Cronbach's Alpha}

\begin{tabular}{|c|rrrrrrr|}
\hline Questions & Q3 & Q4 & Q5 & Q6 & Q7 & Q8 & Q9 \\
\hline Mean & 5.9500 & 5.8500 & 5.6500 & 5.3500 & 5.3750 & 4.9500 & 5.4000 \\
Std Dev & 1.0610 & 1.1886 & 1.1668 & 0.9487 & 0.9524 & 1.2999 & 0.9282 \\
Alpha & 0.9320 & 0.9319 & 0.9428 & 0.9408 & 0.8392 & 0.9260 & 0.8707 \\
\hline
\end{tabular}




\subsection{Correlation and Regression Analysis}

Correlation analysis was used to determine the interrelationships between variables, and with the results presented in Table 6 . The findings show that all variables are significantly related to other variables by applying Pearson's rule of thumb that relationships between variables $>.40$ represents a strong positive relationship and > .70 a very strong positive relationship. The largest variable relationship is between personal-based and Institutional-based trust at 0.7746 and carries a probability of likelihood at $99 \%$. The variable relationships range from 0.5067 to 0.7746 with probability of likelihood from $95-99 \%$. This result also implies that multicollinearity is not a problem. If the variable relationships were close to 1 or -1 , the concern is that the regression coefficient could become unstable resulting in unreliable statistical outcomes (Bryman \& Cramer, 1994). When two variables are highly correlated, they are basically measuring the same phenomena.

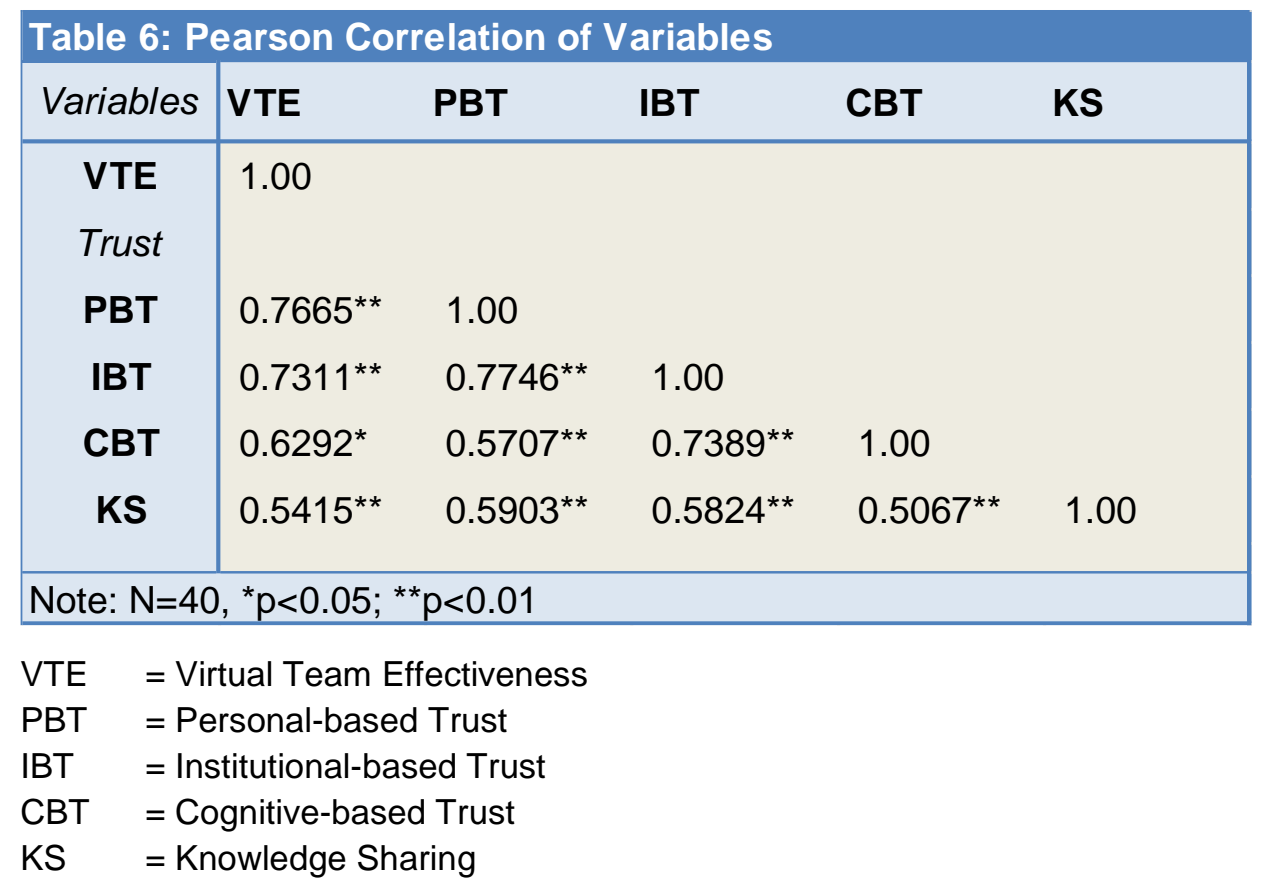

Regression analysis is used to test the hypotheses to gain an understanding of which independent variables are closely related to the dependent variable and to provide a 
basis to explore the form of these relationships. This provides insight into which variables act as predictors of influence on other variables.

The assumptions for multiple regression including normality, linearity, constant variance of the error terms and independent of the error terms have been met, increasing the reliability of the data from the survey. The results of this analysis are presented in Table 7. The regression analysis was also rerun against the company and customer virtual team segmentation to assess the potential effect of these environments by comparing the team member trust relationship. These results are presented in Tables 7a and 7b in Appendix 3.

Regression assumes that the variables have normal distributions. Non-normally distributed variables (highly skewed or variables with substantial outliers) can distort relationships and significance tests. Multiple regression analysis can only accurately estimate the relationship between dependent (DV) and independent variables (IV) if the relationships are linear in nature. If the relationship is non-linear, this risks overestimation (Type I errors) of other IV's that share variance with the IV (Osborne and Waters, 2002). These assumptions were checked from visual data plots and tests validated from the Wessa statistical analysis package outputs (Wessa, 2013).

The multiple regression analysis in Table 7 summarizes the consolidated survey responses. These results identify that all the three areas of trust, personal-based trust, institutional-based trust and cognitive-based trust are significant predictors of virtual team effectiveness and explain $62 \%$ of the variance in virtual team effectiveness. A segmented view of these results based on company and customer virtual team environments is available in Appendix 3 in Tables $7 \mathrm{a}$ and $7 \mathrm{~b}$. 


\begin{tabular}{|cllll|}
\hline \multicolumn{5}{|c|}{ Table 7: Results of Multiple Regression Analysis } \\
\hline Consolidated & H1 & H2 & H3 & H4 \\
\hline DV & VTE (B) & KS (B) & VTE (B) & VTE with KS (B) \\
IV & & & & \\
Trust & & & & \\
PBT & $0.4475^{* *}$ & $0.2723^{*}$ & & $0.5490^{* * *}$ \\
IBT & 0.1788 & 0.1489 & & \\
CBT & 0.2212 & 0.1657 & & \\
& & & & \\
KS & & & $0.6190^{* * *}$ & \\
R & 0.6525 & 0.4006 & 0.2933 & 0.5853 \\
Adj R & 0.6236 & 0.3507 & 0.2747 & 0.5744 \\
F-value & $22.54^{* * *}$ & $8.02^{* * *}$ & $15.77^{* * *}$ & $53.62^{* * *}$ \\
N $=40$ & & & & \\
Note: ${ }^{*} \mathrm{p}<0.05 ;{ }^{* *} \mathrm{p}<0.01 ;{ }^{* * *} \mathrm{p}<0.001$ & & \\
\hline
\end{tabular}

Based on this analysis, $\mathrm{H} 1$ is partially supported with trust being identified as a significant predictor of virtual team effectiveness, however, personal-based trust and not cognitive-based trust presented the strongest correlation relationship.

The results also indicate that only personal-based trust is a significant predictor of knowledge sharing and it also presented the strongest correlation relationship, supporting $\mathrm{H} 2$.

The results highlighted that knowledge sharing is a significant predictor of virtual team effectiveness $(B=0.619, p<0.001)$ and this supports $H 3$.

In testing the mediation effect of knowledge sharing on the relationship between trust and virtual team effectiveness, only personal-based trust is included because institutional-based and cognitive-based trust are not significant predictors of knowledge sharing. Knowledge sharing is a partial mediator of this relationship but the results show that a higher percentage of variance $(58 \%)$ in virtual team effectiveness is being accounted for with knowledge sharing. $\mathrm{H} 4$ is therefore partially supported. 


\subsection{Summary of Hypotheses}

$\mathrm{H} 1$ There is a significant positive relationship Partially between trust and virtual team effectiveness, with Supported cognitive-based trust presenting the strongest correlation.

H2 There is a significant positive relationship Supported between trust and knowledge sharing, with personal-based trust presenting the strongest correlation

H3 There is a significant positive relationship Supported between knowledge sharing and virtual team effectiveness.

H4 Knowledge sharing mediates the relationship Partially between trust and virtual team effectiveness. Supported 


\section{Chapter 5: Discussion and Implications}

This chapter interprets and discusses the data analysis from the responses obtained through the survey and using logic seeks to apply this to the real world of organizations engaging virtual teams. This research provides some interesting insights into the factors that positively influence virtual team effectiveness. The trust relationship is at the heart of this research and the empirical data provides a basis for discussing the structures and interventions that organizations could employ to increase the effectiveness and performance of their virtual teams,

The purpose of the study is to examine the relationship between trust and virtual team effectiveness.

Trust: The approach taken analysed trust from three dimensions (i.e. personalbased, institutional-based and cognitive-based) in an attempt to identify the aspects of the trust relationship that had the most significance in achieving virtual team effectiveness. Knowledge sharing was then assessed in the context of the trust relationship, seeking to understand how prominent it was in enhancing virtual team effectiveness. The study agrees with previous findings (Sarker, Valacich and Sarker, 2003; Pangil and Chan, 2014), where trust was identified as an important factor in achieving virtual team effectiveness. However, this study places a greater weighting on personal-based trust as the dimension of trust that drives the strength of the trust relationship. Personal-based trust focuses on the trust between team members and the reliance that can be placed on others. It is about honesty and integrity and respect for others on the team. Building personal-based trust creates an environment where there is a willingness to share knowledge with others and there is an expectation that the trust is mutual and flows both ways. Your team mates 'will have 
your back' and you stand up for each other. It also creates an environment of 'selfpolicing' within the team.

To achieve team success, team members are dependent on each other to complete tasks and projects. This creates a strong bond across the team and is evidenced in the performance and effectiveness of the team. The barriers of not often meeting face-to-face and in some instances of never meeting present a set of quite different challenges to team members and their working environments. This environment requires trust amongst the team members and is the glue that knits the team together to achieve common goals and to be effective. This presents significant challenges for management in needing to ensure that the virtual team environments provide the individual team members with the opportunity to develop the trust of other members, when of course they are often operating with remoteness and distance as obstacles to deal with. The benefits from advancements in technology have provided greater accessibility and functionally rich communications platforms. This has created better transparency in the operation of virtual teams and accountability of team members to deliver on their commitments. The feedback loop for performance is now often almost instantaneous whereas traditional face-to-face meetings require planning and organization to facilitate times for meetings. Acknowledgement of achievements and the timely dealing with issues through the use of such communications platforms can produce instant results and satisfaction.

Knowledge Sharing: In addition to trust, knowledge sharing is also found to be significantly related to virtual team effectiveness and this is similar to previous findings (Wu, Lin, and Lin, 2006). Knowledge sharing is fundamental to learning and in team environments the pooling of knowledge provides teams with the opportunity to efficiently and effectively achieve their objectives. The total is often greater than 
the sum of the parts. It is the collective knowledge and energy of the team bounded by the trust that the team members have in each other that produces effective teams. Prior research has highlighted that trust between individuals is integral for them to be comfortable and confident to share knowledge with other team members (Abrams, Cross, Lesser, and Levin, 2003; Staples and Webster, 2008; Pangil and Chan, 2014). Knowledge sharing in a virtual environment presents an additional challenge often due to the remoteness of team members. However, collaboration through the use of technology continues to advance and in many organizations the use of communication and collaboration technologies has become so pervasive that they are now using these in preference to face-to-face meetings.

Trust as a psychological state is prone to change (like many other things in life) and this study agrees with earlier research concluding that the effect of trust on virtual team effectiveness is highly complex. The complexity seems to stem from the different types of trust having different effects on virtual team effectiveness. Personalbased and institutional-based trust are shown to both be strongly correlated with virtual team effectiveness, however it is personal-based trust that presents as having a greatest impact on virtual team effectiveness and is strongly supported through knowledge sharing. The earlier study by Pangil and Chan (2014) supports this finding and concludes that cognitive-based trust is not necessary for knowledge sharing. They argued that this was probably true because cognitive-based trust is underpinned by cognitive reasoning and built by individuals' perceptions and interests on the cues of the other party, and it is achieved through frequent and direct interactions with others. The challenge in the virtual team environment is that communications are generally determined by emails, voice and conference calling, denying team members an opportunity to have frequent and direct interactions. Knowledge sharing is of course still active within virtual environments or else teams 
would not achieve their objectives. Cognitive based-trust is also found to be significantly related to virtual team effectiveness and this is consistent with previous findings (Kanawattanachai and Yoo, 2007), inferring that trust is built of individuals' perceptions or interest of the team members and this has a strong impact on the effectiveness of virtual teams. The complexity of trust and its influence on team member interaction strongly suggests that all three dimensions need to be considered by organizations wanting to implement virtual teams and achieve virtual team effectiveness.

This study has identified that personal-based trust and institutional-based trust are both significantly related to knowledge sharing and that the trust that develops because of the personality of the team members and what is institutionalised within the company are key elements to making team members share knowledge with each other. Whilst this supports the argument that trust positively impacts on virtual team effectiveness, it is the personal-based trust identified as the driver for team members to share knowledge and as a consequence, enhance virtual team effectiveness. This differs from the Pangil and Chan (2014) study that found both personal-based trust and institutional-based trust were significant predictors of knowledge sharing.

Personal-based trust is foundered on a judgement that the other person is deemed to be trustworthy or not and it is on this premise that individuals would make the decision to share knowledge. One might expect knowledge to be shared with trustworthy individuals and not with those that are not trusted. Hence, this study has found personal-based trust to be a strong direct influence on virtual team effectiveness via knowledge sharing.

Institutional-based trust is also seen as a significant influence on virtual team effectiveness and especially where there is good organizational structure supporting 
the team and bounded by a strong culture supporting the virtual team environment. The challenge to the organization is that of maintaining regular company communications and interactions with the virtual team members ensuring that they feel part of the team and are all working to achieve the same positive outcomes.

Trust is identified as the cornerstone to achieving virtual team effectiveness and from an organizational perspective this highlights the need for regular communication with the team members to reinforce the culture and values of the company. Organizations need to be employing current up to date information and communication technologies to facilitate better communication, collaboration and project completion. This will enhance the effectiveness of the team and provide team members with the means to build the personal-based trust with other team members. By increasing the team member interactions this can only enhance knowledge sharing and ultimately enhance virtual team effectiveness.

There is also a range of other factors not part of this study that potentially impact on virtual teams and team members' performance. These factors include commitment, empowerment and leadership and the impact of these factors should not be underestimated in terms of the potential influence on virtual team effectiveness. 


\section{Chapter 6: Conclusion}

This chapter concludes this report with a summary of the key findings and responses to the research question. It relates the findings to the purpose of the research, acknowledges the limitations of this study and gives recommendations for future research.

\subsection{Response to the Research Question}

Virtual teams are built on trust and this research concludes that it is the strength of the trust relationship amongst the team members that provides the foundation for virtual team effectiveness. The willingness of team members to share knowledge with others on the team can be directly attributed to the strength of the trust relationship and with this it enhances virtual team effectiveness.

\subsection{Research Limitations}

The research instrument used was a survey, which has its own limitations. While all care was taken to phrase questions as unambiguously as possible, some may still be open to interpretation.

This research is based on a limited number of responses from the staff of one information technology company. Whilst this is not ideal, there is enough data to gain valuable insights into the influence that trust relationships and knowledge sharing have as contributors to achieving virtual team effectiveness. Virtual teams are becoming more prevalent in the business work environment and for us to gain a better understanding of what is a continually evolving structure, further research is required. 


\subsection{Future Research}

Confirmation of the results could be advanced through surveys that engage a broader audience and validate the results obtained in this study. This could also be combined with qualitative research employing semi-structured interviews, for example, to look more carefully into virtual team makeup, structure and communication channels. There are other factors that could influence the team members and the effective operation of virtual teams that could be explored and these include individual motivation and commitment, empowerment of team members and team leadership. An appreciation of the company cultures in which virtual teams operate may also provide a better understanding of the contributors to virtual team effectiveness.

\subsection{Implications for Management}

The nature and make-up of virtual teams pose additional challenges to organizations due to the remoteness of team members and lack of face-to-face interactions. Building the trust relationship between team members is crucial to the future success of virtual teams. It is important that efforts are made to invest in building trust between team members early on and having a programme in place to continue to nurture and sustain the trust relationship between the team members which enhances the sharing of knowledge.

Strategies for building a trust culture within teams is underpinned by the leadership across the organization. Factors that are likely to influence how trust is portrayed include:

- Leading by example - 'walk the talk' and demonstrate trust within the team.

- Open communications - getting team members to talk to one another in an open and honest way. This can be developed through team building exercises 
and maybe the development of team charters. It is useful to help people understand other people's approaches and insights. This can be achieved through the use of psychometric tools such as Myers-Briggs or Kolbe personality testing, and then discussing and sharing the team member profiles within the team. Understanding how others tend to operate and how they will respond to issues in the work environment promotes trust within the team.

- Developing personal trust amongst team members. Promoting social activities and events for team members outside of the work environment to facilitate building stronger bonds between team members and the sharing of values.

- Encourage collective team accountability and develop a shared vision. This creates a self-policing environment for team performance that is built on trust.

- Visibility of team member expertise and skills, as well as the value that each member contributes to the team.

- Creating team based incentive programmes. This aligns the team performance with how individuals on the team are remunerated. It reinforces a team culture and a self-policing environment.

Trust is a primary factor in how people work together, listen to one another, and build effective relationships. When people believe that they are working for trustworthy organizations, they are willing to invest their time and talents in making a difference. People who feel more connected will invest more of themselves in their work. High trust levels lead to a greater sense of self responsibility, greater interpersonal insight, and more collective action toward achieving common goals.

A lack of trust creates cynicism, doubt, and anxiety and this can lead to negativity and generally low energy levels and productivity. 
The implication for management with having staff operating in virtual teams is that it highlights the need for good communication and feedback channels between the organization and its staff.

The challenges for organizations are to understand what level of trust exists across the team, how this impacts on team effectiveness and to be able to apply interventions when seeking to increase team effectiveness.

Active and regular communications programmes, internal marketing campaigns and short surveys are approaches for developing and enhancing the trust relationship.

The trust relationship between team members and sharing of knowledge have both been identified as predictors of virtual team effectiveness and trust as a significant contributing factor in team members sharing knowledge.

One of the best ways to build a sense of trust in people is by sharing information. Sharing information sometimes means disclosing information that is considered privileged, including sensitive and important topics such as the competition's activities, future business plans and strategies, financial data, industry issues or problem areas, competitor's best practices, the way group activities contribute to organizational goals and performance feedback. Providing people with more complete information communicates trust and a sense of "we're in this together." It helps people think more broadly about the organization and the inter-relationships of various groups, resources, and goals

The key to achieving effective virtual teams is how well organizations can implement systems and processes to support nurturing and developing trust amongst the team members of virtual teams. Consideration should be given to team building programmes that engage the team members (ideally face-to-face when establishing 
virtual teams) and work on building trust between the team members. The personalities of team members within virtual teams is also an important factor and achieving the right mix or balance of team members will be a factor in successfully achieving effective virtual teams.

Organizations that are operating multiple virtual teams have the benefit of measuring performance and assessing effectiveness across these teams, and this presents the opportunity to determine and benchmark 'the model virtual team' as well as cycle team members in and out of teams, enabling virtual teams to be refreshed. 


\section{References}

Abrams, L.C., Cross, R., Lesser, E. and Levin, D. (2003), "Nurturing interpersonal trust in knowledge sharing networks", Academy of Management Executive, Vol. 17 No. 4, pp. 64-77.

Arnold, J. A., Arad, S., Rhoades, J. A., \& Drasgow, F. (2000). The empowering leadership questionnaire: The construction and validation of a new scale for measuring leader behaviors. Journal of Organizational Behavior, 21, 249269.

Baron, R. M., \& Kenny, D. A. (1986). The moderator-mediator variable distinction in social psychological research: Conceptual, strategic, and statistical considerations. Journal of personality and social psychology, 51(6), 1173.

Berry, G. R. (2011). Enhancing Effectiveness on Virtual Teams. The Journal of Business Communication, 48(2), 186.

Bryman, A., \& Cramer, D. (1994). Quantitative data analysis for social scientists. London: Routledge.

Burke, K., Aytes, K., \& Chidambaram, L. (2001). Media effects on the development of cohesions and process satisfaction in computer-supported workgroups: An analysis of results from two longitudinal studies. Information Technology and People, 122-141.

Chhay, R. V., \& Kleiner, B. H. (2013). Effective communication in virtual teams. Industrial Management, 55(4), 28-30.

Chiravuri, A., \& Nazareth, D. L. (2011). Cognitive conflict and consensus generation in virtual teams during knowledge capture: Comparative effectiveness of techniques. Journal of Management Information Systems, 28(1), 311-350.

Cohen, S. G., \& Gibson, C. B. (2003). In the beginning: Introduction and framework. Virtual teams that work: Creating conditions for virtual team effectiveness, 1 , 13.

Cohen, S.G. and Bailey, D.E. (1997), "What makes teams work? Group effectiveness research from the shop floor to the executive suite", Journal of Management, Vol. 23, pp. 239-290. 
Cordery, J. L., \& Soo, C. (2008). Overcoming impediments to virtual team effectiveness. Human Factors and Ergonomics in Manufacturing, 18(5), 487500 .

Davenport, T.H. and Prusak, L. (2000), Working Knowledge, 2nd ed., Harvard Business School Press, Boston, MA.

DeRosa, D. (2009), "Virtual success - the keys to effectiveness in leading from a distance", Leadership in Action, Vol. 28 No. 6, pp. 9-11.

DeRosa, D. M., Hantula, D. A., Kock, N., \& D’Arcy, J. (2005). Trust and leadership in virtual teamwork: A media naturalness perspective. Human Resource Management, 43, 219-232.

Driscoll, M. (2000). Psychology of Learning for Instruction. Needham Heights, MA, Allyn \& Bacon.

Dube, L., \& Robey, D. (2008). Surviving the paradoxes of virtual teamwork. Information Systems Journal, 19, 3-30.

Dustdar, S. (2004). Caramba: A process aware collaboration system supporting ad hoc and collaborative processes in virtual teams. Distributed and Parallel Databases, 15, 45-66.

Flanagin, A. J., \& Waldeck, J. H. (2004). Technology use and organizational newcomer socialization. Journal of Business Communication, 41, 137-165.

Gaan, N. (2012). Collaborative tools and virtual team effectiveness: An inductively derived approach in india's software sector. Decision, 39(1), 5-27.

Gera, S., Aneeshkumar, G. S., Fernandez, S. P., Gireeshkumar, G. S., Nze, I. C., \& Eze, U. F. (2013) Virtual teams versus face to face teams: A review of literature. IOSR Journal of Business and Management. Volume 11, Issue 2 (May. - Jun. 2013), 1-4.

Gibson, C. B., \& Cohen, S. G. (Eds.). (2003). Virtual teams that work: Creating conditions for virtual team effectiveness. John Wiley \& Sons.

Gibson, C. B., \& Gibbs, J. L. (2006). Unpacking the concept of virtuality: The effects of geographic dispersion, electronic dependence, dynamic structure, and 
national diversity on team innovation. Administrative Science Quarterly, 51, 451-495.

Griffith, T. L., \& Neale, M. A. (2001). Information processing in traditional, hybrid, and virtual teams: From nascent knowledge to transactive memory. Research in Organizational Behavior, 23, 379-421.

Hackman, J. R. (2002). Leading teams: Setting the stage for great performance. Boston, MA: Harvard Business School Press.

Hakkinen, P. (2004). What makes learning and understanding in virtual teams so difficult? CyberPsychology and Behavior, 7, 201-206.

Hambley, L., O'Neill, T., \& Kline, T. (2007). Virtual team leadership: The effects of leadership styles and communication medium on team interaction styles and outcomes, Organizational Behavior and Human Decision Processes , 10(1), $1-20$.

Hertel, G., Geister, S., \& Konradt, U. (2005). Managing virtual teams: A review of current empirical research. Human Resource Management Review, 15(1), 69-95.

Hinds, P. J., \& Weisband, S. P. (2003). Knowledge sharing and shared understanding in virtual teams. Virtual teams that work: Creating conditions for virtual team effectiveness, 21-36.

Hoegl, M., \& Siebdrat, F. (2009). How to manage virtual teams. MIT Sloan Management Review, 50(4), 63-68.

Holste, J.S. and Fields, D. (2010), "Trust and tacit knowledge sharing and use", Journal of Knowledge Management, Vol. 14 No. 1, pp. 128-140.

Osborne, Jason \& Elaine Waters (2002). Four assumptions of multiple regression that researchers should always test. Practical Assessment, Research \& Evaluation, 8(2). Retrieved October 10, 2014 from http://PAREonline.net/getvn.asp?v=8\&n=2.

Johnson, S. D., Chanidprapa, S., Yoon, S. W., Berrett, J. V., \& LaFleur, J. (2003). Team development and group processes of virtual learning teams. Computers and Education, 39, 379-393. 
Kanawattanachai, P. and Yoo, Y. (2007), "The impact of knowledge coordination on virtual team performance over time”, MIS Quarterly, Vol. 31 No. 4, pp. 783808.

Kirkman, B. L., \& Mathieu, J. E. (2005). The dimensions and antecedents of team virtuality. Journal of Management, 31: 700-718.

Klein, J. A., \& Kleinhanns, A. (2003). Closing the time gap in virtual teams. Creating conditions for effective virtual teams, 381-399.

Lin, C., Standing, C. and Liu, C. (2008), "A model to develop effective virtual teams", Decision Support Systems, Vol. 45 No. 4, pp. 1031-1045.

Lurey, J. S., \& Raisinghani, M. S. (2001). An empirical study of best practices in virtual teams. Information \& Management, 38(8), 523-544.

Mathieu, J. E., Gilson, L. L, \& Ruddy, T. M. (2006). Empowerment and team effectiveness: An empirical test of an integrated model. Journal of Applied Psychology, 91, 97-108.

Maynard, M. T. (2006, August). Group potency: Are virtual teams at a developmental disadvantage? Paper presented at the Academy of Management Conference, Hawaii.

Moshowitz, A.1997, "Virtual Organization", Communications of the ACM, 40(9):3037.

Nonaka, I. (1994), “A dynamic theory of organizational knowledge creation”, Organization Science, Vol. 5, pp. 14-37.

Nonaka, I., \& Toyama, R. (2005). The theory of the knowledge creating firm: Subjectivity, objectivity, and synthesis. Industrial and Corporate Change, 13, 419-436.

Nunamaker Jr, J. F., Reinig, B. A., \& Briggs, R. O. (2009). Principles for effective virtual teamwork. Communications of the ACM, 52(4), 113-117.

Oakley, J.G. (1998). Leadership processes in virtual teams and organizations. The Journal of Leadership Studies, 5, 3-15. 
Pangil, F., \& Chan, J. M. (2014). The mediating effect of knowledge sharing on the relationship between trust and virtual team effectiveness. Journal of Knowledge Management, 18(1), 92-106.

Powell, A., Piccoli, G., \& Ives, B. (2004). Virtual teams: a review of current literature and directions for future research. ACM Sigmis Database, 35(1), 6-36.

Ross, T., Jones, E.C. and Adams, S.G. (2008), "Can team effectiveness be predicted?", Team Performance Management, Vol. 14 Nos 5/6, pp. 248-268.

Salanova, M., Agut, S., \& Peiro, J. M. (2005). Linking organizational resources and work engagement to employee performance and customer loyalty: The mediation of service climate. Journal of Applied Psychology, 90, 1217-1227.

Sarker, S., Valacich, J. S., \& Sarker, S. (2003). Virtual team trust: Instrument development and validation in an IS educational environment. Information Resources Management Journal (IRMJ), 16(2), 35-55.

Staples, D. S., \& Webster, J. (2008). Exploring the effects of trust, task interdependence and virtualness on knowledge sharing in teams. Information Systems Journal, 18(6), 617-640.

Suchan, J., \& Hayzak, G. (2001). The Communication Characteristics of Virtual Teams: A Case Study. IEEE Transactions on Professional Communication, 44(3), 174186The Economist (2010). And the winner is.... The Economist, 396, 79-80.

Vlaar, P. (2008). Co Creating Understanding and Value in Distributed Work. MIS Quarterly, 32, 227-255.

Vroman, K., \& Kovachich, J. (2002). Computer-mediated interdisciplinary teams: Theory and reality. Journal of Interprofessional Care, 16, 159-170.

Wageman, R., Gardner, H., \& Mortensen, M. (2012). The changing ecology of teams: New directions for teams research. Journal of Organizational Behavior, 33(3), 301-315.

Walther, J. B. (1995). Related aspects of computer-mediated communication: Experiential observations. Organizational Science, 6, 180-203. 
Wessa, P. (2013), Multiple Regression (v1.0.29) in Free Statistics Software (v1.1.23r7), Office for Research Development and Education, URL http://www.wessa.net/rwasp multipleregression.wasp/.

Wu, S., Lin, C.S. and Lin, T.C. (2006), "Exploring knowledge sharing in virtual teams: a social exchange theory perspective", Proceedings of the 39th Annual Hawaii International Conference on System Sciences, pp. 1-10.

Xiao, Y-C. and Jin, Y-H. (2010), "The hierarchical linear modeling of shared mental model on virtual team effectiveness", Kybernetes, Vol. 39 No. 8, pp. 13221329.

Yoo, Y. and Alavi, M. (2004), "Emergent leadership in virtual teams. What do emergent leaders do?", Information and Organization, Vol. 14 No. 1, pp. 2758.

Zhang, Y., Fang, Y., Wei, K.K. and Chen, H. (2010), "Exploring the role of psychological safety in promoting the intention to continue sharing knowledge in virtual communities", International Journal of Information Management, Vol. 30 No. 5, pp. 425-436. 


\section{Appendices}

\section{Appendix 1 - Sample Invitation Letter}

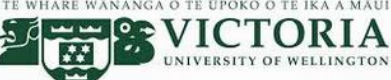

\section{Qual $\amalg=$}

WELCOME

Virtual Team Effectiveness Survey

Are trust and knowledge sharing major contributors to achieving virtual team effectiveness?

Introduction

This survey is part of a Victoria University of Wellington MBA Business Research Project and is focused on exploring the trust relationships between team members so as to better understand how we achieve effectiveness in Virtual Teams.

Ted Thomas, an ex CEO of a Wellington based software company and the researcher has had first hand recent experience in the management of global virtual teams and with rapid advancements in technology, globalisation and the de-centralisation of businesses, he understands the significant challenges faced by management in providing leadership to and communication with members of their teams.

Ted is seeking to validate and extend earlier research into the factors that contribute to team effectiveness and to be able to provide feedback to the management of the company in support of the practices adopted in managing virtual teams operating across the company.

Trust has been found to be a significant predictor of virtual team effectiveness and this research is aimed at reinforcing these earlier findings, and to explore the strength that different types of trust have on team effectiveness and the role of knowledge sharing in mediating the relationship between trust and virtual team effectiveness.

\section{Invitation}

This is a personal invitation to you to participate in the anonymous survey alongside your colleagues. The survey comprises a series of questions designed to gauge your level of agreement or disagreement with proposed statements and you can expect that it will take between 15-20 mins to complete.

No data is being collected that can identify you or any of your colleagues as a respondent. The objective of the survey is to receive an honest assessment of how you believe the virtual teams that you have worked in at the company perform in relation to team effectiveness, team member trust and the sharing of knowledge.

\section{Benefits}

There are no direct benefits for participants. However, it is expected that through your participation, a better understanding will be achieved about how trust and knowledge sharing contribute to virtual team effectiveness. A summary report with the aggregated data will be provided to management and made available to everyone invited to participate in the survey.

This should provide managers and team leaders with information to support the implementation of better management and business practices in achieving virtual team effectiveness.

\section{Confidentiality}

All data obtained from participants will be kept confidential and will only be reported in an aggregate format (by reporting only the combined results). All questionnaires will be anonymous, and no one other than Ted Thomas and his university Supervisor will have access to them. The data collected will be stored in the HIPPA-compliant, Qualtrics-secure database until it is deleted by Ted Thomas (by 31 December 2014).

\section{Participation}

Participation in this research study is completely voluntary.

\section{Questions about the Research}

If you have questions regarding this study, you may contact Ted Thomas, on mob: 0121500582 or email him at ted.thomas@hampton.co.nz or DrPaul McDonald, his supervisor at the Victoria School of Management at Victoria University, PO Box 600, Wellington, ph: 044635506.

Thank you for agreeing to participate in this Research. It is most appreciated. 


\section{Appendix 2 - Survey Questions}

\section{Question 1. Degree of Virtuality}

The First Step is to: Assess your Degree of Virtuality.

This is the approximate amount of time that you work in a Virtual Team.

(In the context of the survey this represents the time you spend working from home and/or at

customer sites. And if working in the office the collaboration time with other remote team members).

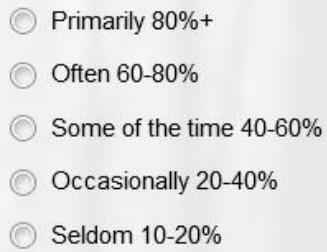

\section{Question 2. Virtual Team}

and to Determine the Nature of your Virtual Team Assignments.

Most of the time I work in:

(1) A Company Test Team

A Customer Test Team (with Customer Team members)

\section{Question 3. Measuring Virtual Team Effectiveness.}

We are then interested in Team Effectiveness - the assessed overall performance of the team and the level of satisfaction of the team members.

Please rate the extent to which you agree or disagree with the following statements:

In the past, my team has been effective in
reaching its goals.
My team is currently meeting its business
objectives.
When my team completes its work, it is
generally on time.
When my team completes its work, it is
generally within the budget.
There is respect for individuals in my team.
I feel the members of my team value my input.
Team member morale is high in my team.
I enjoy being a member of this team.
In the future, I would be interested in
participating in another virtual team.




\section{Question 4. Measuring Personal-based Trust.}

\section{Personal-based Trust}

(as seen in individual's everyday interaction with others - Trustworthy team members)

Please rate the extent to which you agree or disagree with the following statements:

\begin{tabular}{l|l}
$\begin{array}{l}\text { My team members will tell the truth about the } \\
\text { limits of their knowledge. }\end{array}$ \\
$\begin{array}{l}\text { My team members can be counted on to do } \\
\text { what they say they will do. }\end{array}$ \\
$\begin{array}{l}\text { My team members will be honest in describing } \\
\text { their experience and abilities. }\end{array}$
\end{tabular}

\section{Question 5. Measuring Institutional-based Trust.}

\section{Institutional-based Trust}

(as seen in individual's conformity to the rules and regulations of the organisation - Self-monitoring team members)

Please rate the extent to which you agree or disagree with the following statements:

My team members will all do their best because their managers expect that they will always give their best effort

My team members will do their share of the work because we have always been told that in a group project, members should divide and share the work among team members.

My team members will complete deliverables on time because it is known that in their work, delay in completion will lead to negative issues for the team.

My team members will all do their best because their managers expect that they will always give their best effort.

I can depend on my team members because they are my cohorts, and cohorts in an organization are always dependable.

My team members put in their best when it comes to team projects/deliverables.

I can depend on my team members because they will do their best to uphold the reputation of our company.

$\begin{array}{ccccc}\text { Strongly } & \text { Somewhat } & \text { Somewhat } & \text { Strongly } \\ \text { Disagree } & \text { Disagree } & \text { Disagree } & \text { Neutral } & \text { Agree }\end{array}$




\section{Question 6. Measuring Cognitive-based Trust.}

Message based stereotyping - based upon the messages exchanged by team members.

Please rate the extent to which you agree or disagree with the following statements:

From the contents of audio conference or skype meetings, I can understand that my team members are excited about our work.

From the contents of e-mail messages, I can understand that my team members are excited about our work

From the tone of audio conference or skype meetings, I can understand that my team members are excited about our work

From the tone of audio conference or skype meetings, I can understand that my team members are serious about our work

From the frequency of e-mail messages, I can understand that my team members are excited about our work.

From the frequency of e-mail messages, I can understand that my team members are serious about our work.

From the speed of response to email messages, I can understand that my team members are excited about our work.

From the speed of response to email messages, I can understand that my team members are serious about our work

The communications from my team members are mature and professional

\begin{tabular}{|c|c|c|c|c|c|}
\hline & & Sor & $\begin{array}{c}\text { Neither } \\
\text { Agree nor }\end{array}$ & Som & \\
\hline isa & Disagree & Disagree & Disagree & Agree & Agree \\
\hline
\end{tabular}

\section{Question 7. Measuring Cognitive-based Trust.}

Behaviour based stereotyping - based on perceptions of physical appearance and behaviors of team members.

Please rate the extent to which you agree or disagree with the following statements: video conferencing) and seem excited about the project.

My team members are humorous and enthusiastic, and seem excited about working together.

My team members are serious and seem to take the assignment in a serious light.

My team members are dependable because soon after the introduction, our communication focused on how we would tackle the project.

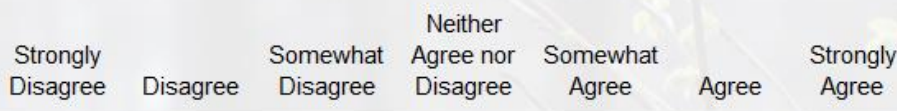




\section{Question 8. Measuring Cognitive-based Trust.}

Reputation based stereotyping - based on the trust placed in team members with good reputations.

Please rate the extent to which you agree or disagree with the following statements:

I can depend on my team members because I
have heard that they are always committed to
their work.
I can depend on my team members because I
have heard of their excellent performance in
previous projects.
$\begin{aligned} & \text { My team members are more experienced than I } \\ & \text { am and can be depended upon. } \\ & \text { My team members seem more organised than I } \\ & \text { am and hence can be depended on. }\end{aligned}$

\section{Question 9. Measuring the effect of Trust on Knowledge Sharing.}

And lastly we are interested in the Sharing of Knowledge by Team Members

Please rate the extent to which you agree or disagree with the following statements:

People in this team keep their best ideas to
themselves.
People in this team are willing to share
knowledge/ideas with others in this team.
People in this team share their ideas openly.
People in this team with expert knowledge are
willing to help others in this team.
This team is good at using the knowledge/ideas of
team members.




\section{Appendix 3 - Company and Customer Virtual Team Segmentation}

Table 7a represents analysis of the results from those respondents operating in company virtual teams. With only 14 responses, this sample was too small to provide any significant insights into how the trust relationships influenced team effectiveness of company virtual teams.

\begin{tabular}{|cllll|}
\hline \multicolumn{5}{|c|}{ Table 7a: Results of Multiple Regression Analysis } \\
Company & H1 & H2 & H3 & H4 \\
\hline DV & VTE (B) & KS (B) & VTE (B) & VTE with KS (B) \\
IV & & & & \\
Trust & & & & 0.0300 \\
PBT & 0.1124 & 0.1011 & & \\
IBT & -0.0112 & 0.0899 & & \\
CBT & 0.4888 & 0.0899 & & \\
& & & -0.2115 & \\
KS & & & 0.0674 & 0.3073 \\
$R^{2}$ & 0.4266 & 0.0774 & 0.0000 & 0.2496 \\
Adj R & 0.2546 & 0.0000 & 0.0000 & $5.32^{* * *}$ \\
F-value & 2.48 & 0.28 & 0.87 & \\
N=14 & & & & \\
Note: ${ }^{*} \mathrm{p}<0.05 ;{ }^{* *} \mathrm{p}<0.01 ;{ }^{* * *} \mathrm{p}<0.001$ & & \\
\hline
\end{tabular}

Table $7 \mathrm{~b}$ represents the analysis of the results from those respondents operating in customer virtual teams and with a larger number of responses, this analysis shows that all three areas of trust, personal-based trust, institutional-based trust and cognitive-based trust are significant predictors of virtual team effectiveness, with these types of trust explaining $69 \%$ of variance in virtual team effectiveness for staff operating within customer virtual teams. 


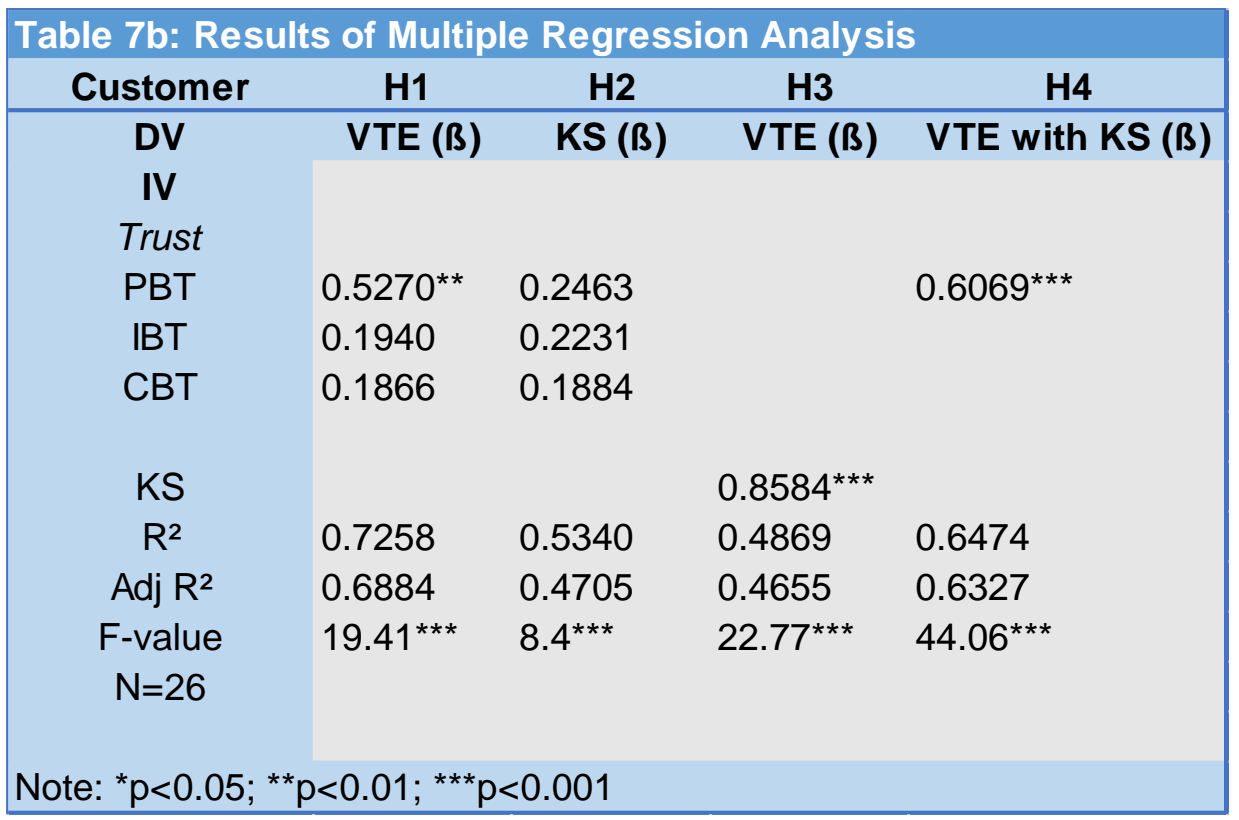

Responses from those staff operating in customer virtual teams, show knowledge sharing standing out as a significant predictor of virtual team effectiveness, explaining $86 \%$ of the variance in virtual team effectiveness. Personal-based trust is also a significant predictor of virtual team effectiveness when mediated by knowledge sharing.

The weighting of responses from staff that work within Customer virtual teams $(65 \%)$ could be the reason why institutional-based trust did not feature as prominently as it did in earlier research as a significant predictor of virtual team effectiveness. It could be that these team members associate themselves more closely with the culture and institution of the customer than that of the employer organization. This is a risk that organizations are faced with when the staff align themselves more closely with the customer virtual team and its structures. To overcome this risk, organizations need to implement staff rotation policies and strengthen the communications with staff to reinforce the company values and culture. 


\section{Glossary}

Adjusted $\boldsymbol{R}^{2}$ is an attempt to take account of the phenomenon of the $R^{2}$ automatically and spuriously increasing when extra explanatory variables are added to the model. It is a modification due to that adjusts for the number of explanatory terms in a model relative to the number of data points. The adjusted $R^{2}$ can be negative, and its value will always be less than or equal to that of $R^{2}$. Unlike $R^{2}$, the adjusted $R^{2}$ increases when a new explanatory variable is included only if the new explanatory variable improves the $R^{2}$ more than would be expected by chance. If a set of explanatory variables with a predetermined hierarchy of importance are introduced into a regression one at a time, with the adjusted $R^{2}$ computed each time, the level at which adjusted $R^{2}$ reaches a maximum, and decreases afterward, would be the regression with the ideal combination of having the best fit without excess/unnecessary terms.

Cronbach's $\alpha$ (alpha) is a coefficient of internal consistency. It is commonly used as an estimate of the reliability of question items. A commonly accepted rule for describing internal consistency using Cronbach's alpha is as follows, however, a greater number of items in the test can artificially inflate the value of alpha and a sample with a narrow range can deflate it:

\begin{tabular}{|l|l|}
\hline Cronbach's alpha & Internal consistency \\
\hline$\alpha \geq 0.9$ & Excellent \\
\hline $0.7 \leq \alpha<0.9$ & Good \\
\hline $0.6 \leq \alpha<0.7$ & Acceptable \\
\hline $0.5 \leq \alpha<0.6$ & Poor \\
\hline$\alpha<0.5$ & Unacceptable \\
\hline
\end{tabular}

$\mathbf{F}$ value is a statistic that tests the overall significance of the regression model. Specifically, they test the null hypothesis that all of the regression coefficients are equal to zero. This tests the full model against a model with no variables and with the estimate of the dependent variable being the mean of the values of the dependent variable. The $\mathrm{F}$ value is the ratio of the mean regression sum of 
squares divided by the mean error sum of squares. Its value will range from zero to an arbitrarily large number.

Pearson's Correlation is a measure of the linear correlation (dependence) between the variables, giving a value between +1 and -1 inclusive, where 1 is total positive correlation, 0 is no correlation, and -1 is total negative correlation.

$p$-value is the probability of obtaining a test statistic result at least as extreme or as close to the one that was actually observed, assuming that the null hypothesis is true. A researcher will often "reject the null hypothesis" when the $p$-value turns out to be less than a predetermined significance level, often 0.05 or 0.01 . Such a result indicates that the observed result would be highly unlikely under the null hypothesis.

$\boldsymbol{R}^{2}$ is a statistic that will give some information about the goodness of fit of a model. In regression, the $R^{2}$ coefficient of determination is a statistical measure of how well the regression line approximates the real data points. An $R^{2}$ of 1 indicates that the regression line perfectly fits the data. 